\title{
Similarity Between the Mandelbrot Set and Julia Sets
}

\section{TAN Lei ${ }^{\star}$}

Institut für Dynamische Systeme, Universität Bremen, W-2800 Bremen 33, Federal Republic of Germany

Received July 10, 1989

\begin{abstract}
The Mandelbrot set $M$ is "self-similar" about any Misiurewicz point $c$ in the sense that if we examine a neighborhood of $c$ in $M$ with a very powerful microscope, and then increase the magnification by a carefully chosen factor, the picture will be unchanged except for a rotation. The corresponding Julia set $J_{c}$ is also "self-similar" in the same sense, with the same magnification factor. Moreover, the two sets $M$ and $J_{c}$ are "similar" in the sense that if we use a very powerful microscope to look at $M$ and $J_{c}$, both focused at $c$, the structures we see look like very much the same.
\end{abstract}

\section{Introduction}

For a quadratic polynomial $f_{c}: z \mapsto z^{2}+c$, the filled-in Julia set $K_{c}$ of $f_{c}$ is the set of non-escaping points under iteration:

$$
K_{c}=\left\{z \in \mathbb{C} \mid\left(f_{c}^{n}(z)\right)_{n \in \mathbb{N}}, \text { is bounded }\right\},
$$

where $f_{c}^{n}$ denotes the $n^{\text {th }}$ iteration $f_{c} \circ f_{c} \circ \ldots \circ f_{c}$ of $f$. The Julia set of $f_{c}$ is $J_{c}=\partial K_{c}$.

The Mandelbrot set is

$$
M=\left\{c \in \mathbb{C} \mid 0 \in K_{c}\right\} .
$$

One can generate easily the pictures of Julia sets and the Mandelbrot set by computers. Figure 1 is a picture of $M$, Fig. 3a-3d are pictures of $J_{c}$ for various values of $c$. Globally, $J_{c}$ and $M$ have completely different shapes. However, their local structures are sometimes very similar. Figure 2 consists of three successive enlargements of $M$ in a neighborhood of $i$. A remarkable resemblance with the Julia set for $c=i$ (Fig. 3a) appears. In fact, this kind of similarity happens for every value of $c$ which is a Misiurewicz point, that is, for which the point 0 under $f_{c}$ is not

*. Present address: Ecole Normale Supérieure de Lyon, 46 Allée d'Italie, F-69364 Lyon, France 
Fig. 1
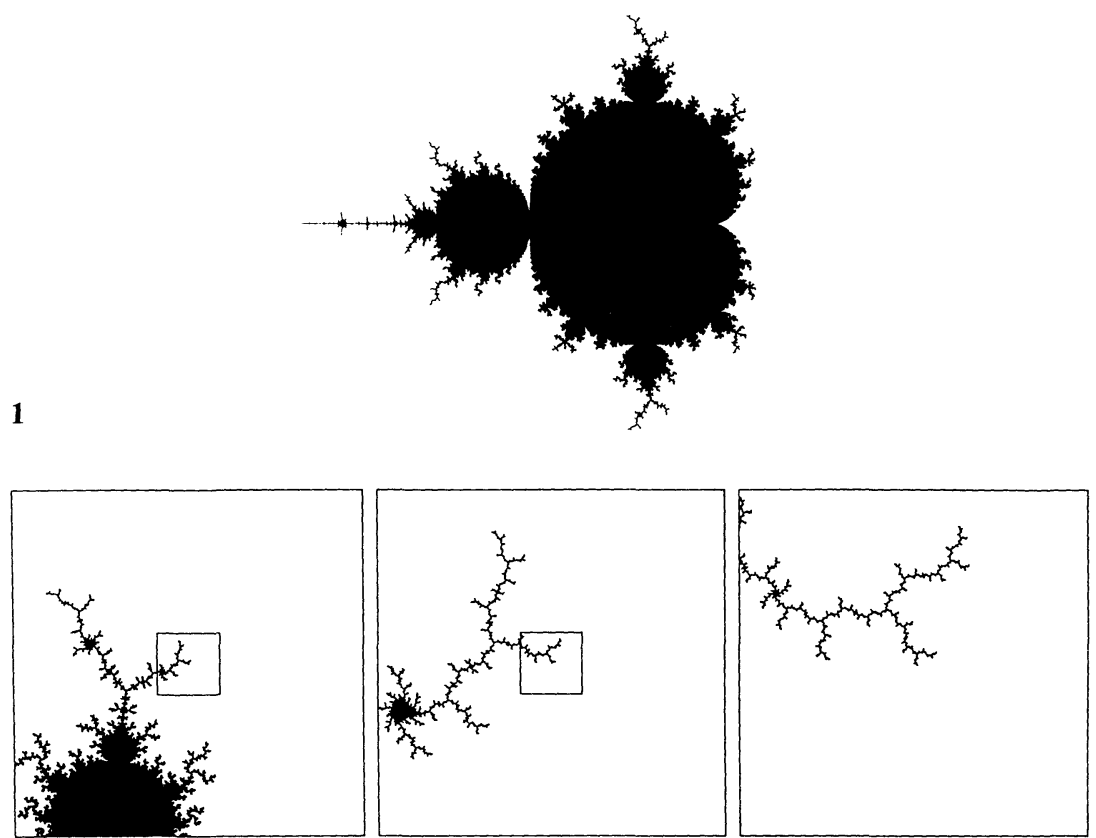

Fig. 2. Three successive magnifications of $M$ as indicated by the inserted frames. Center: $i$. Width of the first picture: $0.5656854 \approx 0.1 \times 4 \sqrt{2}$. Magnification factor: $4 \sqrt{2}$
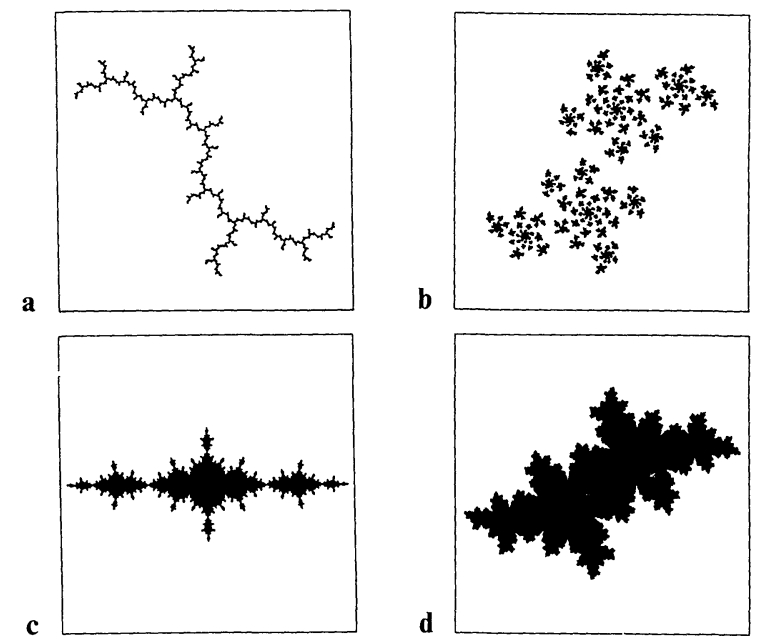

Fig. 3a-d. The Julia set $J_{c}$. a $c=i$. Center: 0 , width: 3.0 . b $c=0.11031-0.67037 i$. Center: 0 , width: 3.0. $c=-1.25$. Center: 0 , width: 3.6. $\mathbf{d} c=-0.481762-0.531657 i$. Center: 0 , width: 3.0

periodic but falls eventually into a periodic orbit. The main topic of this paper is to analyse this similarity.

The point $c=i$ is one of the simplest examples of Misiurewicz points: for $f_{i}: z \mapsto z^{2}+i$, the orbit of 0 is: $0 \mapsto i \mapsto i-1 \mapsto-i \mapsto i-1$. We have $f_{i}^{4}(0)=f_{i}^{2}(0)$. The point $c=-2$ is another one: for $f_{-2}: z \mapsto z^{2}-2$, the orbit of 0 is: $0 \mapsto-2 \mapsto 2 \mapsto 2$. In 
Sect. 5 we will give further examples. Using the Montel theorem, one can show that the set of Misiurewicz points forms a countable dense subset of the boundary of $M$ [M].

In this paper, we will show that for every Misiurewicz point $c$, a powerful magnification of $J_{c}$ focused at $c$ and the same magnification of $M$ focused also at $c$ will give very similar structures (up to a multiplication by a complex number), and the more powerful the magnification is, the more similar the structures are. We say that $J_{c}$ and $M$ are asymptotically similar about $c$. Moreover, both $J_{c}$ and $M$ are asymptotically self-similar about $c$ in the sense that if we increase successively the magnifications to $J_{c}$ (respectively $M$ ) focused at $c$ by a carefully chosen factor, what we see are more and more the same.

Section 2 consists of definitions of asymptotic similarity and asymptotic selfsimilarity, and introduces the Hausdorff-Chabauty distance in order to compare two closed sets asymptotically. Section 3 discusses the asymptotic self-similarity of any Julia $J_{c}$ about any repulsive periodic point, and about any point which is eventually repulsive periodic. This can be applied immediately to the case where $c$ is a Misiurewicz point, since the point $c$ is eventually repulsive periodic under $f_{c}$. Section 4 contains a key proposition which makes connections of the selfsimilarity between parameter space and dynamical spaces. This proposition is stated in a high-dimension form, in order to be applied eventually to other topics. Section 5 shows finally the asymptotic similarity between $M$ and $J_{c}$ about $c$ for $c$ a Misiurewicz point, by applying the result of Sect. 4 . Theorem 5.5 is a review of the principal steps of the whole proof. At the end of Sect. 5 we calculate some specific examples. Section 6 consists of some related results, and a discussion of the similarity problems about other points of $M$.

The main part of this work was done in 1984, under the direction of Professor A. Douady. A preliminary version of this article ([T2]) was inserted in [DH2].

The author would like to thank A. Douady for his introduction and his powerful support of this work; without him this work would not have existed. I also thank J. H. Hubbard, M. Shishikura, Y. Fisher, B. Bielefeld, and B. Branner for their helpful discussions: some of the calculations are made directly by $\mathbf{M}$. Shishikura. I am indebted to H.-O. Peitgen and his group in Bremen for hospitality and great interest in the work.

\section{Hausdorff Distance and Asymptotic Similarity}

In this section we discuss the definition of asymptotic similarities of closed subsets of $\mathbb{C}$ and some immediate results.

Definition 1 (Hausdorff distance). Denote by $\mathscr{F}$ the set of non-empty compact subsets of $\mathbb{C}$. For $A, B \in \mathscr{F}$, we define the semi-distance from $A$ to $B$ to be

$$
\delta(A, B)=\sup _{x \in A} d(x, B)
$$

and the Hausdorff distance of $A, B$ to be

$$
d(A, B)=\sup (\delta(A, B), \delta(B, A)) .
$$

This distance makes $\mathscr{F}$ a complete metric space. By the definition, $\delta(A, B)<\varepsilon$ signifies that $A$ is contained in the $\varepsilon$-neighborhood of $B$. 
The next definition is for comparing two closed subsets of $\mathbb{C}$ within a bounded region.

Definition 2. For $r$ a positive real number, we denote by $D_{r}$ the disc centered at 0 of radius $r$. For any closed set $A \subset \mathbb{C}$, we define a compact set

$$
A_{r}=\left(A \cap \overline{D_{r}}\right) \cup \partial D_{r} \text {. }
$$

(It is for a technical reason that we add $\partial D_{r}$ to $A \cap \bar{D}_{r}$.) Or, in order to analyse the local behavior of $A$ near a point $a \in A$, we use the translation $\tau_{-a}: z \mapsto z-a$ to translate $a$ to the origin and define

$$
\left(\tau_{-a} A\right)_{r}=\left(\left(\tau_{-a} A\right) \cap \bar{D}_{r}\right) \cup \partial D_{r}
$$

For $A, B$ two closed sets in $\mathbb{C}$, we have $A_{r}, B_{r} \in \mathscr{F}$. We can then define the Hausdorff-Chabauty distance of $A, B$ in the window of $D_{r}$ to be (Fig. 4)

$$
d_{r}(A, B)=\sup \left(\delta\left(A_{r}, B_{r}\right), \delta\left(B_{r}, A_{r}\right)\right) \text {. }
$$

Definition 3. Assume $\varrho=|\varrho| e^{i \theta},|\varrho|>1,0 \leqq \theta<2 \pi$.

1. A closed subset $B$ of $\mathbb{C}$ is $\varrho$-self-similar about 0 if there is $r>0$ such that

$$
(\varrho B)_{r}=B_{r} .
$$

$B$ is also considered to be self-similar about 0 with the scale $\varrho$. This says that under the magnification with the factor $|\varrho|$ together with the rotation of angle $\theta$ the set $B$ remains unchanged within $\bar{D}_{r}$.

2. A closed subset $A$ of $\mathbb{C}$ is asymptotically $\varrho$-self-similar about a point $x \in A$ if there is $r>0$ and a closed set $B$ such that

$$
\left(\varrho^{n} \tau_{-x} A\right)_{r} \rightarrow B_{r} \quad \text { while } n \rightarrow \infty
$$

for the Hausdorff distance. The set $B$ is automatically $\varrho$-self-similar about 0 , it is called the limit model of $A$ at $x$.

3. Two closed sets $A$ and $B$ are asymptotically similar about 0 if there is $r>0$ such that

$$
\lim _{t \in \mathbb{C}, t \rightarrow \infty} d_{r}(t A, t B)=0
$$
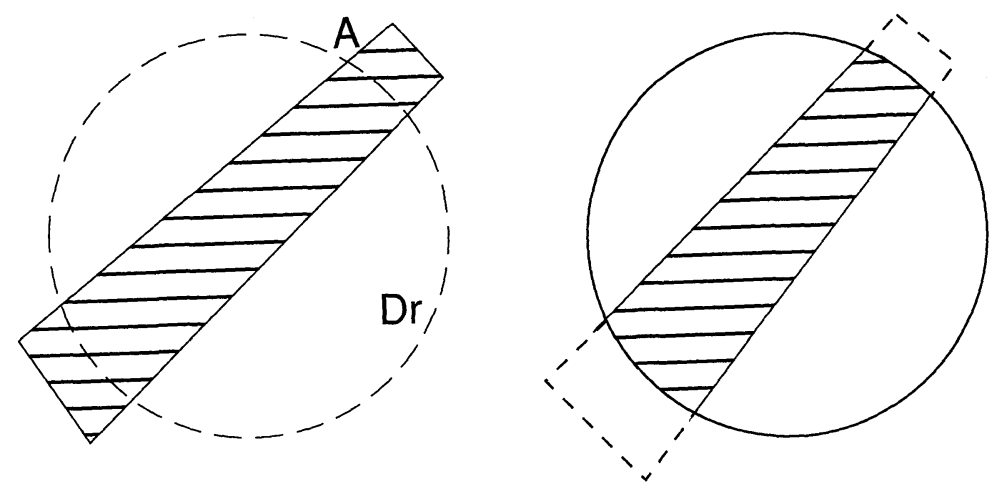

Fig. 4. $A$ and $A_{r}$ 
Remark. The property of similarity does not depend really on the value of $r$. In fact if (2), (3), or (4) hold for some value $r>0$, then they hold for any $0<\tau^{\prime} \leqq \tau$, and (4) holds for every $r>0$, since $t \rightarrow \infty$. Moreover, if $B$ satisfies (2) for some value $r>0$, we can set

$$
B^{\prime}=\bigcup_{n \in \mathbb{N}} \varrho^{n}\left(B_{r}\right)
$$

Then $B^{\prime} \cap \bar{D}_{r}=B \cap \bar{D}_{r}$ and $\left(\varrho B^{\prime}\right)_{s}=B_{s}^{\prime}$ for any $s>0$. If $A, B$ satisfy (3) for some value $r>0$, then by replacing $B$ by $B^{\prime}$, the equality (3) will hold for every value $s>0$ instead of $r$ (since $|\varrho|^{n} \rightarrow \infty$ while $n \rightarrow \infty$ ).

\section{Examples}

1. The disc $\bar{D}_{r}$ itself is trivially self-similar. A line, a segment are self-similar. A curve with a tangent is asymptotically similar to its tangent.

2. The spiral $S(\lambda)=\left\{e^{\lambda x} \mid x \in \mathbb{R}\right\}$ is $e^{\lambda}$-self-similar about 0 if $\left|e^{\lambda}\right|>1$ (Fig. 5).

3. The Cantor set $C$. $C$ is self-similar about any rational point, and is not selfsimilar about any irrational point $x \in C$. Let $x=0 . t_{1} t_{2} t_{3} \ldots$ be the expansion of $x \in C$ in base three. Then each $t_{i}$ is either 0 or $2 . x$ is rational if and only if this expansion is eventually periodic, i.e. there are $l$ and $p$ such that $t_{n}=t_{n+p}$ for each $n \geqq l$. The minimal $p$ is the eventual period of $x$. Hence $C$ is $3^{p}$-self-similar at $x \in C$ if and only if $x$ is rational and the eventual period of $x$ divides $p$.

Lemma 2.1. Assume $A, B$ closed. Fix $r>0$. We have

1. For every $a \in A \cap \bar{D}_{r}, d\left(a, B_{r}\right) \leqq d(a, B) \leqq d\left(a, B \cap \bar{D}_{r}\right)$.

2. $\delta\left(A_{r}, B_{r}\right) \leqq \sup _{a \in A \cap \bar{D}_{r}} d(a, B) \leqq d\left(A \cap \bar{D}_{r}, B \cap \bar{D}_{r}\right)$.

Proof. Left to the reader.

\section{Proposition 2.2.}

1. For $A, B$ closed subsets of $\mathbb{C}$, if there are $r>0, \varrho,|\varrho|>1$ such that

$$
\lim _{n \rightarrow \infty} d_{r}\left(\varrho^{n} A, \varrho^{n} B\right)=0
$$

then

$$
\lim _{t \rightarrow \infty, t \in \mathbb{C}} d_{r}(t A, t B)=0,
$$

i.e. $A$ and $B$ are asymptotically similar about 0 .
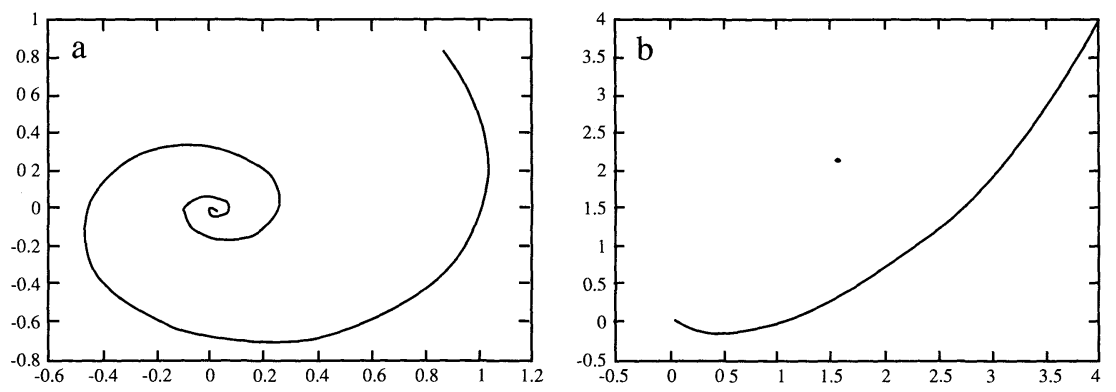

Fig. 5. a $e^{(\log 1.2+\pi i / 4) x} ; \mathbf{b} e^{(\log (4 \sqrt{2})+\pi i / 4) x}$ 
2. Any asymptotically self-similar set is asymptotically similar to its limit model.

\section{Proof.}

1. For each $\varepsilon>0$, there is $N>0$ such that if $n>N$ then $\delta\left(\left(\varrho^{n} A\right)_{r},\left(\varrho^{n} B\right)_{r}\right)<\varepsilon$. If $|t|>T$ $=|\varrho|^{N}$ then $|\varrho|^{n} \leqq|t|<|\varrho|^{n+1}$ for some $n>N$. Hence for each $x \in(t A) \cap \bar{D}_{r}$ we have $x=t a=\left(t / \varrho^{n}\right) \varrho^{n} a$ with $a \in A$ and

$$
\left|\varrho^{n} a\right| \leqq r \frac{|\varrho|^{n}}{|t|} \leqq r .
$$

Hence $\varrho^{n} a \in\left(\varrho^{n} A\right) \cap \bar{D}_{r}$. From

$$
\delta\left(\left(\varrho^{n} A\right)_{r},\left(\varrho^{n} B\right)_{r}\right)<\varepsilon
$$

we get

$$
d\left(\varrho^{n} a,\left(\varrho^{n} B\right)_{r}\right)<\varepsilon .
$$

Since $|t| \geqq|\varrho|^{n}$, we have

$$
\frac{t}{\varrho^{n}}\left(\varrho^{n} B\right)_{r} \cap \bar{D}_{r}=\left(\frac{t}{\varrho^{n}} \varrho^{n} B\right) \cap \bar{D}_{r},
$$

hence

$$
\left(\frac{t}{\varrho^{n}}\left(\varrho^{n} B\right)_{r}\right)_{r}=\left(\frac{t}{\varrho^{n}} \varrho^{n} B\right)_{r}
$$

So

$$
\begin{aligned}
d\left(x,(t B)_{r}\right) & =d\left(\frac{t}{\varrho^{n}} \varrho^{n} a,\left(\frac{t}{\varrho^{n}} \varrho^{n} B\right)_{r}\right)=d\left(\frac{t}{\varrho^{n}} \varrho^{n} a,\left(\frac{t}{\varrho^{n}}\left(\varrho^{n} B\right)_{r}\right)_{r}\right) \\
& \leqq d\left(\frac{t}{\varrho^{n}} \varrho^{n} a, \frac{t}{\varrho^{n}}\left(\varrho^{n} B\right)_{r}\right) \leqq \frac{|t|}{\left|\varrho^{n}\right|} d\left(\varrho^{n} a,\left(\varrho^{n} B\right)_{r}\right)<\frac{|t|}{|\varrho|^{n}} \varepsilon<|\varrho| \varepsilon,
\end{aligned}
$$

hence

$$
d\left(x,(t B)_{r}\right)=d\left(t a,(t B)_{r}\right)<|\varrho| \varepsilon .
$$

According to the above lemma,

$$
\delta\left((t A)_{r},(t B)_{r}\right) \leqq \sup _{x \in(t A) \cap \bar{D}_{r}} d\left(x,(t B)_{r}\right)<|\varrho| \varepsilon .
$$

This proves $\delta\left((t A)_{r},(t B)_{r}\right) \rightarrow 0$ while $t \rightarrow \infty$. A similar analysis will give also $\delta\left((t B)_{r},(t A)_{r}\right) \rightarrow 0$ while $t \rightarrow \infty$.

2. Let $A$ be asymptotically $\varrho$-self-similar about 0 with the limit model $B$. Fixe $r>0$, with $(\varrho B)_{r}=B_{r}$. Then $\left(\varrho^{n} B\right)_{r}=B_{r}$ and

$$
\lim _{n \rightarrow \infty} \delta\left(\left(\varrho^{n} A\right)_{r},\left(\varrho^{n} B\right)_{r}\right)=\lim _{n \rightarrow \infty} \delta\left(\left(\varrho^{n} A\right)_{r}, B_{r}\right)=0
$$

and

$$
\lim _{n \rightarrow \infty} \delta\left(\left(\varrho^{n} B\right)_{r},\left(\varrho^{n} A\right)_{r}\right)=\lim _{n \rightarrow \infty} \delta\left(B_{r},\left(\varrho^{n} A\right)_{r}\right)=0 .
$$

$A, B$ verify (6) and then (7) by part 1. 
Proposition 2.3. Suppose that $U, V$ are two neighborhoods of 0 in $\mathbb{R}^{2}$ and $F: U \rightarrow V$ is a $\mathscr{C}^{1}$ diffeomorphism with $F(0)=0$ and with the derivative $T_{0} F=T$ non-singular. Then for any closed set $A \subset U$ the two sets $T A$ and $F(A)$ are asymptotically similar about 0 , i.e. for some $r>0$ (in fact for every $r>0$ )

$$
\lim _{t \in \mathbb{C}, t \rightarrow \infty} D_{r}(t F(A), t T A)=0 .
$$

Proof. Fix $r>0$. For all $\varepsilon>0$, then exists $\delta>0$ such that if $<\|x\|<\delta$ then

$$
\frac{\|F(x)-T x\|}{\|x\|}<\varepsilon
$$

For each $y \in t T A \cap \bar{D}_{r}$, there is $x \in A$ such that $y=t T x$ with $\|x\| \leqq\left|t^{-1}\right| \cdot\left\|T^{-1}\right\| r$ $=C /|t|$, where $C=\left\|T^{-1}\right\| r$ is a constant.

So for $|t|>N=C / \delta$ and any $y=t T x \in(t T A) \cap \bar{D}_{r}, y \neq 0$ we have $0<\|x\|<\delta$ and

$$
\begin{aligned}
d(t T x, t F(A)) & \leqq|t| \cdot\|F(x)-T x\| \leqq \frac{C}{|t| \cdot\|x\|}|t| \cdot\|F(x)-T x\| \\
& =C \frac{\|F(x)-T x\|}{\|x\|}<C \varepsilon .
\end{aligned}
$$

Then by Lemma 2.1

$$
\delta\left((t T A)_{r},(t F(A))_{r}\right) \leqq \sup _{y \in(t T A)_{\cap} \cap \bar{D}_{r}} d(y, t F(A))<C \varepsilon .
$$

A similar analysis will give $\delta\left((t F(A))_{r},(t T A)_{r}\right) \rightarrow 0$.

If $F(A)$ is asymptotically self-similar then $T A$ is so too. If in addition $T$ commutes with the similarity scale, $A$ should be also asymptotically self-similar. More precisely,

Proposition 2.4. Assume that $F$ is as in Proposition 2.3. Suppose that $A$ is a closed set such that $F(A)$ is asymptotically $\varrho$-self-similar about 0 with the limit model $B$. If $T$ is $\mathbb{C}$-linear, then $A$ is asymptotically $\varrho$-self-similar about $x$, and the limit model is $T^{-1} B$, i.e. there is $r^{\prime}>0$ such that

$$
\lim _{n \rightarrow \infty} d_{r^{\prime}}\left(\varrho^{n}\left(\tau_{-x} A\right), T^{-1} B\right)=0 .
$$

Proof. By the above proposition, $T A$ and $F(A)$ are similar about 0 . Choose $r>0$ such that

$$
\lim _{n \rightarrow \infty} d_{r}\left(\varrho^{n} T A, \varrho^{n} F(A)\right)=0
$$

and

$$
\lim _{n \rightarrow \infty} d_{r}\left(\varrho^{n} F(A), B\right)=0
$$

Then

$$
\lim _{n \rightarrow \infty} d_{r}\left(\varrho^{n} T A, B\right)=0 \text {. }
$$

Since $T$ is $\mathbb{C}$-linear, we have $\varrho^{n} T A=T \varrho^{n} A$. For $r^{\prime}>0$ such that $D_{r^{\prime}} \subset T^{-1} D_{r}$,

$$
\lim _{n \rightarrow \infty} d_{r^{\prime}}\left(\varrho^{n} A, T^{-1} B\right)=0 .
$$


This proposition will be our essential tool in the next section to prove the selfsimilarity of Julia sets. The next proposition gives another way to produce new self-similar sets:

Proposition 2.5. Suppose that $X$ is a (asymptotically) @-self-similar set about 0 . Then the set

$$
Y=\left\{z \in \mathbb{C} \mid z^{d} \in X\right\}
$$

is also (asymptotically) $\varrho$-self-similar about 0 . In fact for any $\varrho^{\prime}$ such that $\varrho^{\prime d}=\varrho$, the set $Y$ is (asymptotically) $\varrho^{\prime}$-self-similar about 0.

Proof. We will only deal with the self-similar case. Choose $r>0$ such that $(\varrho X)_{r^{d}}=X_{r^{d}}$. Remark at first

$$
\begin{gathered}
Y \cap D_{r}=\left\{z \in \mathbb{C} \mid z^{d} \in X \cap D_{r^{d}}\right\}, \\
z \in Y \cap D_{r /\left|\varrho^{\prime}\right|} \Leftrightarrow z^{d} \in X \cap D_{r^{d} /\left|\varrho^{d}\right|} \Leftrightarrow \varrho z^{d} \in X \cap D_{r^{d}} \\
\end{gathered}
$$

Hence $\left(\varrho^{\prime} Y\right) \cap D_{r}=Y \cap D_{r}$ and then $\left(\varrho^{\prime} Y\right)_{r}=Y_{r}$.

\section{Self-similarity of Julia Sets}

In this section we discuss the self-similarity of the Julia set of rational maps. And we apply the result to the case of Misiurewicz points.

Definition and known results (cf. [D] and [DH1]).

1. Let $f: \overline{\mathbb{C}} \rightarrow \overline{\mathbb{C}}$ be a rational map. A point $x \in \overline{\mathbb{C}}$ is periodic of period $p$ if $p$ is the minimal number such that $f^{p}(x)=x$. The value $\varrho=\left(f^{p}\right)^{\prime}(x)$ is called the eigenvalue of the orbit of $x$, it does not depend on $x$, but only on the orbit of $x$. A periodic point $x$ is said to be

(a) repulsive if $|\varrho|>1$,

(b) attractive if $0<|\varrho|<1$,

(c) superattractive if $\varrho=0$,

(d) rational indifferent if $\varrho=e^{2 \pi i \theta}$, with $\theta$ rational,

(e) irrational indifferent if $\varrho=e^{2 \pi i \theta}$, with $\theta$ irrational.

2. A point $x \in \mathbb{C}$ is eventually periodic if there are integers $l \geqq 0$ and $p \geqq 1$ such that $f^{p}\left(f^{l}(x)\right)=f^{l}(x)$. We say that $x$ is eventually repulsive (attractive, ..., etc.) periodic if $f^{l}(x)$ is repulsive (attractive, ..., etc.).

3. A closed set $A$ is said to be completely invariant under $f$ if $f(A)=f^{-1}(A)=A$. If $A$ is completely invariant under $f$ it is completely invariant under $f^{k}$ as well.

4. The Julia set $J_{f}$ of $f$ is the closure of the set of repulsive periodic points of $f$. We know that $J_{f}$ is completely invariant by $f$.

5. For $P$ a monic complex polynomial, the filled-in Julia set $K_{P}$ of $P$ is

$$
K_{P}=\left\{z \in \mathbb{C} \mid P^{n}(z), n \in \mathbb{N}, \text { is bounded }\right\} .
$$

We have $J_{P}=\partial K_{P}$ and $K_{P}$ is completely invariant under $P$. 
Remark. There are several equivalent definitions of the Julia set. The reason that we choose this one is that repulsive periodic points play the central role in the study of asymptotic similarity.

Concerning Misiurewicz points, we recall

Definition.

1. The family of quadratic polynomials can be parametrized by (up to affine conjugacy)

$$
\left\{f_{c}: z \mapsto z^{2}+c \mid c \in \mathbb{C}\right\} .
$$

$K_{c}$ denotes the filled-in Julia set of $f_{c}$ and $J_{c}$ the Julia set.

2. The Mandelbrot set is

$$
M=\left\{c \in \mathbb{C} \mid 0 \in K_{c}\right\} .
$$

3. A point $c \in M$ is a Misiurewicz point if 0 is eventually periodic for $f_{c}$ but not periodic.

A classical result of Douady and Hubbard shows:

Proportion 3.1 [DH1]. For c a Misiurewicz point,

1. 0 and then $c$ is eventually repulsive periodic;

2. $K_{c}=J_{c}$, i.e. $K_{c}$ has no interior.

Here comes the main theorem of this section:

Theorem 3.2. Let $f$ be a rational map and $A$ be a completely invariant closed set under $f$. Assume that $x$ is an eventually repulsive periodic point for $f$. Then $A$ is asymptotically self-similar about $x$, with the scale $\varrho$ equal to the eigenvalue of the eventual periodic orbit of $x$. There is a conformal mapping $\phi$ defined in a neighborhood $\bar{U}$ of $x$ such that $\frac{1}{\phi^{\prime}(x)} \phi(A \cap \bar{U})$ is the limit model of $A$ at $x$. Moreover, if $x$ is periodic, we can choose $\phi$ such that $\phi^{\prime}(x)=1$; if $x$ is eventually periodic with $f^{p}\left(f^{l}(x)\right)=f^{l}(x)$ and if in addition, $\left(f^{l}\right)^{\prime}(x) \neq 0$, then the limit models of $A$ at $x$ and at $f^{l}(x)$ are the same, up to a multiplication by $\left(f^{l}\right)^{\prime}(x)$.

Hence not only $A$ is asymptotically self-similar about $x$, but its limit model can be realized locally (via a conformal mapping) instead of just asymptotically.

In the case where $c$ is a Misiurewicz point, we can apply this theorem immediately to $A=J_{c}$ about $x=c$ and claim that $J_{c}$ is asymptotically self-similar about $c$, since the point $c$ is eventually repulsive under $f_{c}$. Corollary 3.5 below will give the precise formulas.

To prove Theorem 3.2, we need at first a classical lemma (see, for example, [D]):

\section{Lemma 3.3.}

1. Suppose that $U, V$ are neighborhoods of $x$ in $\mathbb{C}$ and $g: U \rightarrow V$ is a holomorphic function with $g(x)=x$ and $\left|g^{\prime}(x)\right| \neq 0,1$. Set $\varrho=g^{\prime}(x)$. Then $g$ is locally conjugate to its linear part $z \mapsto \varrho z$. Mote precisely, there is a conformal mapping $\varphi$ defined in a neighborhood of $x$ with $\varphi(x)=0, \varphi^{\prime}(x)=1$,

$$
\varphi \circ g \circ \varphi^{-1}(z)=\varrho z .
$$


The explicit form of $\varphi$ is given by

$$
\varphi(z)=\lim _{n \rightarrow \infty} \varrho^{n}\left(\left(g^{-1}\right)^{n}(z)-x\right),
$$

where $\mathrm{g}^{-1}$ is the unique inverse mapping of $g$ defined in a neighborhood of $x$. The mapping $\varphi$ is called the linearization mapping of $g$ about $x$.

2. Suppose that $U, V$ are neighborhoods of $x, y$ in $\mathbb{C}$ respectively and $g: U \rightarrow V$ is holomorphic with $g(x)=y$ and $g^{\prime}(x)=g^{\prime \prime}(x)=\ldots=g^{(d-1)}(x)=0$ and $g^{(d)}(x) \neq 0$. Then there are $U_{x} \subset U, V_{y} \subset V$ neighborhoods of $x, y$ respectively, $s>0$, such that for each conformal mapping $\varphi_{y}: V_{y} \rightarrow D_{s^{d}}$ there is (with $d$ choices) a conformal mapping $\varphi_{x}: U_{x} \rightarrow D_{s}$ satisfying

$$
\varphi_{y} \circ g \circ \varphi_{x}^{-1}(z)=z^{d} .
$$

Lemma 3.4. Assume that $A$ is a completely invariant set of $f$. Then for any $U \subset \overline{\mathbb{C}}$, we have

$$
\begin{gathered}
f(A \cap U)=A \cap f(U), \\
f^{-1}(A \cap f(U)) \cap U=A \cap U .
\end{gathered}
$$

Proof. $f(A \cap U) \subset f(A) \cap f(U)=A \cap f(U)$. On the other hand, $y \in A \cap f(U) \Rightarrow y \in A$ and $y=f(x)$ for some $x \in U$. Since $f^{-1}(A)=A$ we have $x \in A$ and hence $y \in f(U \cap A)$. So we have also $A \cap f(U) \subset f(A \cap U)$,

$$
f^{-1}(A \cap f(U)) \cap U=\left(f^{-1}(A) \cap f^{-1}(f(U))\right) \cap U=A \cap U .
$$

Proof (of Theorem 3.2).

a) Assume at first that $x$ is periodic.

Let $p$ be the period of $x$ and $\varrho=\left(f^{p}\right)^{\prime}(x)$ be the eigenvalue of the orbit of $x$. By the assumption, $|\varrho|>1$. So $f^{p}$ arises to a homeomorphism in a neighborhood of $x$. Remark that $x$ is a fixed point of $f^{p}$. According to Lemma 3.3 we can find a neighborhood $V$ of $x$, a neighborhood $V_{0}$ of 0 , a conformal mapping $\varphi: V \rightarrow V_{0}$ such that $\varphi(x)=0, \varphi^{\prime}(x)=1$ and

$$
\varphi \circ f^{p}(z)=\varrho(\varphi(z))
$$

Take $r>0$ such that $\bar{D}_{r} \subset V_{0}$. Set $U=\varphi^{-1}\left(D_{r / \ell}\right)$ and $B=\varphi(A \cap \bar{U})$. We have $f^{p}(U)=\varphi^{-1}\left(D_{r}\right) \subset V$ and $\bar{U} \subset f^{p}(U)$. Since $A$ is completely invariant under $f^{p}$, from Lemma 3.4, we have $\mathrm{f}^{p}(A \cap U)=A \cap f^{p}(U)$. Hence

$$
f^{p}(A \cap U) \cap U=A \cap U \text {. }
$$

Then

$$
\varphi\left(f^{p}(A \cap U) \cap U\right)=\varphi(A \cap U) .
$$

Since $\varphi$ is bijective, we have

$$
\begin{gathered}
\varphi\left(f^{p}(A \cap U)\right) \cap \varphi(U)=\varphi(A) \cap \varphi(U), \\
\varrho(\varphi(A \cap U)) \cap D_{r / \varrho}=\varphi(A) \cap D_{r / \varrho}, \\
\varrho\left(\varphi(A) \cap D_{r / \varrho}\right) \cap D_{r / \varrho}=\varphi(A) \cap D_{\tau / \varrho},
\end{gathered}
$$

i.e.

$$
\varrho\left(B \cap D_{r / \varrho}\right) \cap D_{r / \varrho}=B \cap D_{r / \varrho} .
$$


This proves $(\varrho B)_{r / \varrho}=B_{r / \varrho}$. Hence $B$ is $\varrho$-self-similar about 0. Apply Proposition 2.4 to $\varphi: V \rightarrow V_{0}, A \cap \bar{U} \rightarrow B$; we conclude that $A$ is asymptotically $\varrho$-self-similar about $x$, and the limit model is exactly

$$
\frac{1}{\varphi^{\prime}(x)} B=B=\varphi(A \cap \bar{U})
$$

b) Assume now that $x$ is eventually periodic.

Let $l, p$ be minimal such that $f^{p}\left(f^{l}(x)\right)=f^{l}(x)$. Set $\alpha=f^{l}(x)$ and $\varrho=\left(f^{p}\right)^{\prime}(\alpha)$. By the assumption, $|\varrho|>1$. Applying the part a) to $\alpha$, we can find a neighborhood $U_{\alpha}$ of $\alpha$, a conformal mapping $\varphi$ such that $\varphi(\alpha)=0, \varphi^{\prime}(\alpha)=1$, the set $X=\varphi\left(A \cap \bar{U}_{\alpha}\right)$ is $\varrho$-self-similar and

$$
\lim _{n \rightarrow \infty} d_{r^{\prime}}\left(\varrho^{n} \tau_{-\alpha} A, X\right)=0
$$

for some $r^{\prime}>0$.

b1) Assume at first that the set $\left\{x, f(x), \ldots, f^{l}(x)\right\}$ does not contain any critical point of $f$, in other words, $\left(f^{l}\right)^{\prime}(x) \neq 0$.

Then $f^{l}$ maps a neighborhood $\bar{U}_{x}$ of $x$ homeomorphically to a neighborhood $\bar{V}_{\alpha} \subset U_{\alpha}$ of $\alpha$, and $f^{l}\left(A \cap \bar{U}_{x}\right)=A \cap \bar{V}_{\alpha}$. Apply Proposition 2.4 to $f^{l}: \bar{U}_{x} \rightarrow \bar{V}_{\alpha}$; we get

$$
\lim _{n \rightarrow \infty} d_{r}\left(\varrho^{n}\left(\tau_{-x} A\right), \frac{1}{\left(f^{l}\right)^{\prime}(x)} X\right)=0
$$

for some $r>0$. The theorem is then done by setting $\phi=\varphi \circ f^{l}$.

b2) Suppose now $\left(f^{l}\right)^{\prime}(x)=\left(f^{l}\right)^{\prime \prime}(x)=\ldots=\left(f^{l}\right)^{(d-1)}(x)=0$ and $\left(f^{l}\right)^{(d)}(x) \neq 0$.

Then by Lemma 3.3 there are $U_{x}, \varphi_{x}, s, 0<s<r^{1 / d}$ such that

$$
\varphi \circ f^{l} \circ \varphi_{x}^{-1}: D_{s} \rightarrow D_{s^{d}}, \quad z \rightarrow z^{d} .
$$

Set

$$
Y=\left\{z \in \mathbb{C} \mid z^{d} \in X\right\}
$$

We have $Y=\varphi_{x}\left(A \cap U_{x}\right)$, since $X=\varphi \circ f^{l}\left(A \cap U_{x}\right)=\left(\varphi_{x}\left(A \cap U_{x}\right)\right)^{d}$. According to Proposition 2.5 the set $Y$ is also $\varrho$-self-similar about 0 . The theorem is then given by the next formula with $\phi=\varphi_{x}$ :

$$
\lim _{n \rightarrow \infty} d_{s}\left(\varrho^{n}\left(\tau_{-x} A\right), \frac{1}{\varphi_{x}^{\prime}(x)} Y\right)=0 .
$$

Proof of (9). Fix $s>0$. We prove first

$$
\delta\left(\left(\varrho^{n}\left(\tau_{-x} A\right)\right)_{s},\left(\frac{1}{\varphi_{x}^{\prime}(x)} Y\right)_{s}\right) \rightarrow 0 .
$$

For $z \in \varrho^{n}\left(\tau_{-x} A\right) \cap \bar{D}_{s}$, we have $z=\varrho^{n}(y-x)$ with $y \in A$ and $|y-x| \leqq s /|\varrho|^{n}$. We can take $n$ large enough such that $y \in A \cap U_{x}$. Then

$$
\begin{aligned}
d\left(z,\left(\frac{1}{\varphi_{x}^{\prime}(x)} Y\right)_{s}\right) & \leqq d\left(z, \frac{1}{\varphi_{x}^{\prime}(x)} Y\right) \leqq\left|\varrho^{n}(y-x)-\frac{\varrho^{n} \varphi_{x}(y)}{\varphi_{x}^{\prime}(x)}\right| \\
& =\left|\varrho^{n}(y-x)\right| \cdot\left|1-\frac{\varphi_{x}(y)}{(y-x) \varphi_{x}^{\prime}(x)}\right| \rightarrow 0
\end{aligned}
$$


while $n \rightarrow \infty$.

A similar analysis will give

$$
\delta\left(\left(\frac{1}{\varphi_{x}^{\prime}(x)} Y\right)_{s},\left(\varrho^{n}\left(\tau_{-x} A\right)\right)_{s}\right) \rightarrow 0,
$$

and then (9).

We are ready now to look at Misiurewicz points:

Corollary 3.5. Assume that $c$ is a Misiurewicz point. Let l, p be the minimal integers such that

$$
f_{c}^{p}\left(f_{c}^{l}(c)\right)=f_{c}^{l}(c)
$$

Set $\alpha=f_{c}^{l}(c), \varrho=\left(f_{c}^{p}\right)^{\prime}(\alpha)$. Then

1. $|\varrho|>1$.

2. There is a conformal mapping $\varphi$ defined in a neighborhood of $\alpha$, with $\varphi(\alpha)=0$, $\varphi^{\prime}(\alpha)=1$ and

$$
\varphi \circ f_{c}^{p} \circ \varphi^{-1}(z)=\varrho z
$$

where $\varphi$ is given by

$$
\lim _{n \rightarrow \infty} \varrho^{n}\left(\left(f_{c}^{-p}\right)^{n}(z)-\alpha\right)
$$

with $f_{c}^{-p}$ the local inverse of $f_{c}^{p}$ in a neighborhood of $\alpha$.

3. $\left(f_{c}^{l}\right)^{\prime}(c) \neq 0$.

4. There are $r>0$, a neighborhood $V$ of $\alpha$, a neighborhood $U$ of $c$, such that $f_{c}^{l}(\bar{U})=\bar{V}$ and

$$
\begin{aligned}
& \lim _{n \rightarrow \infty} d_{r}\left(\varrho^{n}\left(\tau_{-c} J_{c}\right), \frac{1}{\left(f_{c}^{l}\right)^{\prime}(c)} \varphi\left(J_{c} \cap \bar{V}\right)\right) \\
& \quad=\lim _{n \rightarrow \infty} d_{r}\left(\varrho^{n}\left(\tau_{-c} J_{c}\right), \frac{1}{\left(f_{c}^{l}\right)^{\prime}(c)} \varphi \circ f_{c}^{l}\left(J_{c} \cap \bar{U}\right)\right)=0 .
\end{aligned}
$$

Hence $J_{c}$ is asymptotically self-similar about $c$ with the scale $\varrho$ and the limit model $\varphi\left(J_{c} \cap \bar{V}\right)$.

Proof.

1. From Proposition 3.1.

2. The existence of $\varphi$ is guaranteed by Lemma 3.3 if we replace $g$ by $f_{c}^{p}$ and $x$ by $\alpha$. 3. Since 0 is the unique critical point of $f_{c}$ and is not periodic, there is no critical point of $f_{c}$ in

$$
\left\{c, f_{c}(c), \ldots, f_{c}^{l}(c)\right\}
$$

hence $\left(f_{c}^{l}\right)^{\prime}(c) \neq 0$.

4. It is a consequence of parts a) and b1) in the proof of Theorem 3.2, by setting $A=J_{c}$ and $x=c$.

We give now some examples to illustrate the result of Theorem 3.2. 


\section{Examples}

1. Figure $3 b$ is the Julia set for $c=0.11031-0.67037 i$. The polynomial $f_{c}$ has two fixed points, both repulsive. We choose $x=(1-\sqrt{1-4 c}) / 2 \approx-0.14205$ $-0.52205 i$ to be one of them. The period $p$ of $x$ is then 1 , and the eigenvalue $\varrho$ of $x$ is $2 x \approx-0.2841-1.0441 i$. Figure 6 consists of a sequence of magnifications of $J_{c}$ at $x$, the magnification factor from one image to the next is $\varrho^{3}$ (since $|\varrho| \approx 1.082$ is too close to 1$)$.

2. Figure $3 c$ is the Julia set for $c=-1.25$. It has a very different shape from Fig. $3 b$. In fact one is a connected set and the other one is homeomorphic to a Cantor set. But the fixed point $x=(1-\sqrt{1-4 c}) / 2$ which is $(1-\sqrt{6}) / 2 \approx-0.72475$ in this case is again a repulsive fixed point for $f_{c}=f_{-1.25}$. So the self-similar phenomenon occurs again around this point. We have $p=1$ and $\varrho \approx-1.4495$. Figure 7 consists of three magnifications of $J_{c}$ at $x$, the magnification factor from one image to the next is $|\varrho|=1.4495$. Since $\varrho$ is real negative, there is a difference of the rotation of angle $180^{\circ}$ from one image to the next. Figure $7 \mathrm{a}$ and Fig. $7 \mathrm{c}$ then look the same.

3. Let us check the Misiurewicz point $c=i$ now. For the polynomial $f_{i}: z \mapsto z^{2}+i$, the orbit of $i$ is: $i \mapsto i-1 \mapsto-i \mapsto i-1$. The point $i$ is eventually repulsive periodic, with $l=1, p=2, \alpha=f_{c}^{l}(c)=i-1$,

$$
\varrho=\left(f_{c}^{2}\right)^{\prime}(\alpha)=4+4 i=4 \sqrt{2} \mathrm{e}^{\pi i / 4}
$$

and $\left(f_{c}^{l}\right)^{\prime}(c)=\left(f_{i}\right)^{\prime}(i)=2 i$. Denote by $X$ the limit model of $J_{i}$ at $i-1$ and $Z$ the limit model of $J_{i}$ at $i$. By our theorem, we have $Z=(1 / 2 i) X$. Figure $8 \mathrm{a}$ is a magnification of Fig. $3 \mathrm{a}$ in a neighborhood of $i-1$. Figure $8 \mathrm{~b}$ is a magnification of Fig. $8 \mathrm{a}$ with
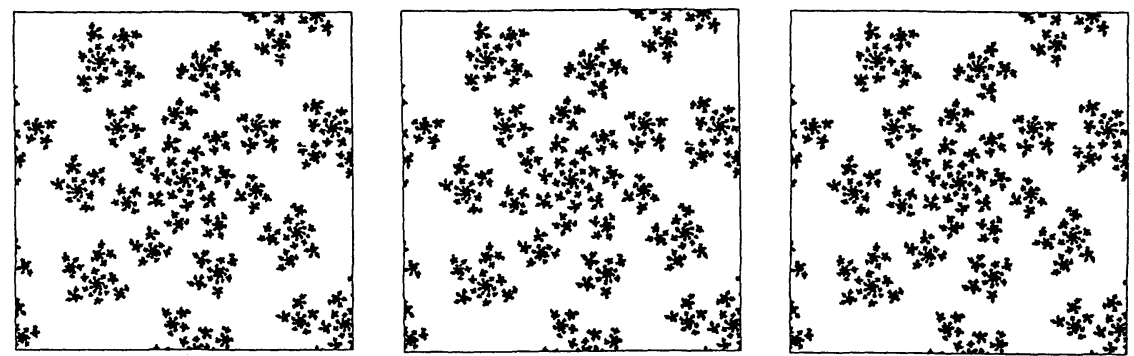

Fig. 6. Three successive magnifications of $J_{\mathrm{c}}$ for $c=0.11031-0.67037 i$. Center: -0.14205 $-0.52205 i$, width of the first picture: 0.01 . Magnification factor: 1.26688 , rotation: $44.3349^{\circ}$
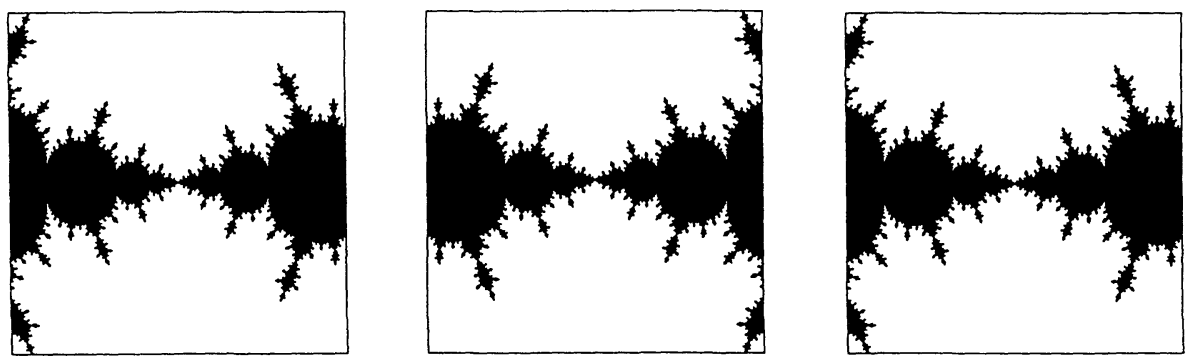

Fig. 7. Three successive magnifications of $J_{c}$ for $c=-1.25$. Center: -0.72475 , width of the first picture: 0.01 . Magnification factor: 1.4495 , rotation: 0 

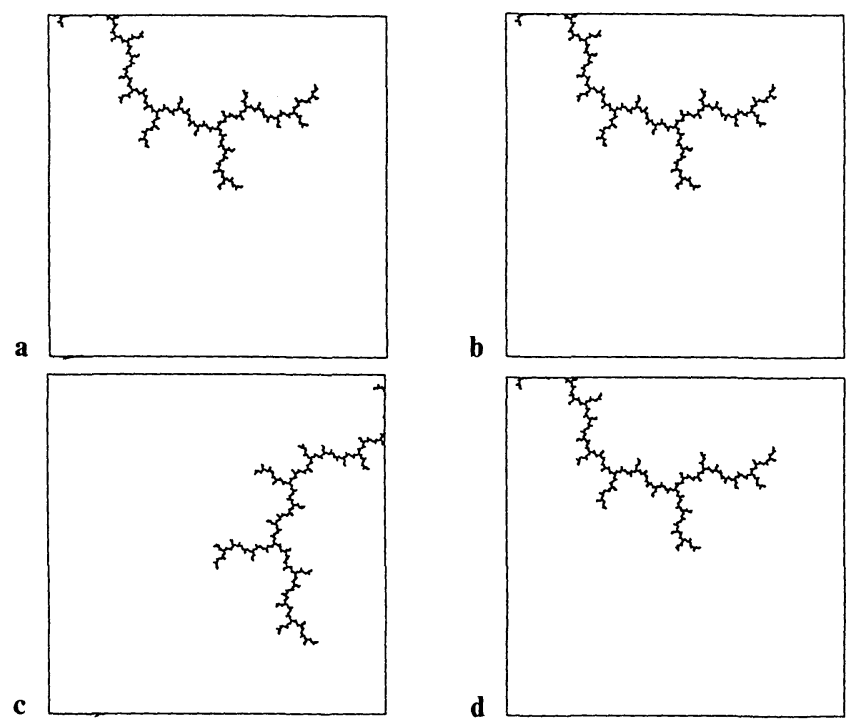

Fig. 8a-d. Magnification of $J_{c}$ for $c=i$. a Center $i-1$, widths: 0.001 , rotation: 0 . b Center $i-1$, width: $0.001 / 4 \sqrt{2}$, rotation: $45^{\circ}$. c Center $i$, with: 0.0005 , rotation: 0 . d Center $i$, width: 0.0005 , rotation: $90^{\circ}$

the factor $4 \sqrt{2} e^{\pi i / 4}$. Figure $8 \mathrm{c}$ is a magnification of Fig. 3a in a neighborhood of $i$ with the width of the window equal to the half of the width in Fig. 8a. Figure $8 \mathrm{~d}$ is Fig. $8 \mathrm{c}$ rotated by an angle of $90^{\circ}$. Compare Figs. $8 \mathrm{a}$ and $8 \mathrm{~d}$, one is centered at $i-1$, the other one is centered at $i$. This computer experiment confirms by impression our result.

\section{Key Proposition}

This section will give a connection between the self-similarities of dynamic spaces and parameter spaces, in a high-dimension form.

It is easy to generalize the notion of similarity to closed subsets of an euclidean space of finite dimension. Let $E=\mathbb{C}^{k}$ and $\mathscr{F}$ be the set of compact subsets of $E$. Denote by $D_{r}$ the ball of $E$ centered at 0 with radius $r$. Then the Hausdorff distance and the Hausdorff-Chabauty distance are defined in exactly the same way. Moreover,

\section{Definition.}

1. A linear mapping $L: E \rightarrow E$ is contracting if there are $\sigma<1$ positive and $C$ positive such that for all $n \in \mathbb{N},\left\|L^{n}\right\| \leqq C \sigma^{n}$. We say that $L$ is expanding if $L$ is invertible and $L^{-1}$ is contracting.

2. A subset $B$ of $E$ is $L$-self-similar about 0 if $L: E \rightarrow E$ is linear expanding and there is $r>0$ such that $(L B)_{r}=B_{r}$. We also say that $B$ is self-similar about 0 with scale $L$. 3. A subset $B$ of $E$ is asymptotically $L$-self-similar about a point $x \in B$ if $L: E \rightarrow E$ is linear expanding and there is $r>0, A \in \mathscr{F}$ such that

$$
\lim _{n \rightarrow \infty}\left(L^{n} \tau_{-x} B\right)_{r}=A
$$


exists for the Hausdorff distance (where $\tau_{\alpha}$ is the translation $z \mapsto z+\alpha$ ). The set $A$ is automatically $L$-self-similar about 0 , it is called the limit model of $B$ at $x$.

Here is our main result of this section:

Proposition 4.1. Suppose $\Delta$ is a neighborhood of $\lambda_{0}$ in $E$, and $X \subset \Delta \times E$. For each $\lambda \in \Delta$, set

$$
X(\lambda)=\{x \in E \mid(\lambda, x) \in X\} .
$$

Let $u: \Delta \rightarrow E$ be a continuous mapping, with $u\left(\lambda_{0}\right)=0$. Set

Assume:

$$
M=M_{u}=\{\lambda \in \Delta \mid u(\lambda) \in X(\lambda)\}
$$

Condition 1. $X$ is closed in $\Delta \times E$.

Condition 2. (Existence of a dense set of continuous sections at $\left(\lambda_{0}, 0\right)$.) There is $A \subset X\left(\lambda_{0}\right)$, dense in $X\left(\lambda_{0}\right)$, such that for each $x \in A$, there exists $U_{x} \subset \Delta$, neighborhood of $\lambda_{0}$, and a continuous mapping $h_{x}: U_{x} \rightarrow E$ with $h_{x}\left(\lambda_{0}\right)=x$ and $h_{x}(\lambda) \in X(\lambda)$.

Condition 3. $X(\lambda)$ is $L(\lambda)$-self-similar about 0 with a fixed radius $r^{\prime}$, and $\lambda \mapsto L(\lambda)$ is continuous, with

$$
\left\|L(\lambda)-L\left(\lambda_{0}\right)\right\|=O(\|\lambda\|)
$$

and

$$
\left\|L\left(\lambda_{0}\right)\right\| \cdot\left\|L\left(\lambda_{0}\right)^{-1}\right\|^{2}<1,
$$

we can assume then for $\lambda \in \Delta$ (by restricting $\Delta$ if necessary),

$$
\|L(\lambda)\| \leqq \mu_{1}, \quad\left\|L(\lambda)^{-1}\right\| \leqq \mu_{2}
$$

and

$$
\mu_{1} \cdot \mu_{2}^{2}=\mu<1
$$

Condition 4. The derivative $S=T_{\lambda_{0}} u$ exists and is non-singular, and

$$
\|u(\lambda)-S \lambda\|=O\left(\|\lambda\|^{2}\right) \text {. }
$$

Then there is $r>0$ such that

a) Under the conditions 1,2 , we have $d_{r}\left(X(\lambda), X\left(\lambda_{0}\right)\right) \rightarrow 0$ when $\lambda \rightarrow \lambda_{0}$.

b) Under the conditions $1,2,3,4$, the set $S \tau_{-\lambda_{0}} M$ is asymptotically $L\left(\lambda_{0}\right)$-self-similar about 0 , and the limit model is $X\left(\lambda_{0}\right)$. Precisely we have

$$
\lim _{n \rightarrow \infty}\left(L\left(\lambda_{0}\right)^{n} \cdot S \tau_{-\lambda_{0}} M\right)_{r}=\left(X\left(\lambda_{0}\right)\right)_{r} .
$$

c) If $S$ and $L\left(\lambda_{0}\right)$ commute, then $M$ is asymptotically $L\left(\lambda_{0}\right)$-self-similar about $\lambda_{0}$, and the limit model is $S^{-1} X\left(\lambda_{0}\right)$, i.e. there is $s>0$,

$$
\lim _{n \rightarrow \infty}\left(L\left(\lambda_{0}\right)^{n} \tau_{-\lambda_{0}} M\right)_{s}=\left(S^{-1} X\left(\lambda_{0}\right)\right)_{s} .
$$

Remark. Equation (12) is automatically verified if $L\left(\lambda_{0}\right)=\varrho I$. Otherwise, let $\left\{l_{1}, l_{2}, \ldots, l_{k}\right\}$ be the set of eigenvalues of $L\left(\lambda_{0}\right)$ and

$$
1<\left|l_{1}\right| \leqq\left|l_{2}\right| \leqq \ldots \leqq\left|l_{k}\right| .
$$


If $\left|l_{k}\right|<\left|l_{1}\right|^{2}$ then there is a norm such that (12) holds. This requires that $L\left(\lambda_{0}\right)$ deforms the centered balls in a somehow homogeneous way. M. Shishikura has a counter-example which shows that the proposition becomes false if the condition (12) is not satisfied.

Condition 1 can be stated in an equivalent but more useful form:

Lemma 4.2. The following two conditions are equivalent:

1. $X$ is a closed subset of $\Delta \times E$;

2. For each $\lambda_{0} \in \Delta$, the set $X\left(\lambda_{0}\right)$ is closed in $E$ and for all $r>0$, and $\lim _{\lambda \rightarrow \lambda_{0}} \delta\left(X_{r}(\lambda)\right.$, $\left.X_{r}\left(\lambda_{0}\right)\right)=0$.

We leave the proof to the reader.

Proof (of Proposition 4.1). Without loss of generality, we may assume $\lambda_{0}=0$.

a) By Condition 1 and Lemma 4.2, fix any $r>0$ we have

$$
\lim _{\lambda \rightarrow 0} \delta\left(X_{r}(\lambda), X_{r}(0)\right)=0 .
$$

Let us prove now

$$
\lim _{\lambda \rightarrow 0} \delta\left(X_{r}(0), X_{r}(\lambda)\right)=0
$$

by using the dense set of continuous sections of the Condition 2. Denote by $V_{r}(\varepsilon)$ the $\varepsilon$-neighborhood of $\partial D_{r}$. Since $X_{r}(0)$ is compact, and $A$ is dense in $X(0)$ by Condition $2, \forall \varepsilon>0$, there is a finite number of points $x_{1}, x_{2}, \ldots, x_{m}$ in $A$ such that

$$
X_{r}(0) \subset V_{r}(\varepsilon / 2) \cup\left(\bigcup_{i=1}^{m} D\left(x_{i}, \varepsilon / 2\right)\right)
$$

where $D\left(x_{i}, \varepsilon / 2\right)$ is the ball centered at $x_{i}$ and with radius $\varepsilon / 2$. Now for each $i, \exists \eta_{i}>0$ small enough such that when $\|\lambda\|<\eta_{i}$, we have

$$
d\left(h_{x_{i}}(\lambda), x_{i}\right)<\varepsilon / 2
$$

so

$$
d\left(x_{i}, X(\lambda)\right) \leqq \varepsilon / 2 .
$$

Hence $\forall \varepsilon>0$, for $\|\lambda\|<\eta=\min \left\{\eta_{i}\right\}, \forall x \in X(0) \cap \bar{D}_{r}$, we have

$$
d\left(x, X_{r}(\lambda)\right) \leqq d(x, X(\lambda)) \leqq\left\|x-x_{i}\right\|+d\left(x_{i}, X(\lambda)\right)<\varepsilon
$$

for some $i$, hence $\delta\left(X_{r}(0), X_{r}(\lambda)\right)<\varepsilon$.

b) Set $M(n)=L(0)^{n} \cdot S M$ and let $r>0$ be smaller than $r^{\prime}$ required in Condition 3 . Our proof consists of two steps:

b1) $\delta\left(M_{r}(n), X_{r}(0)\right) \rightarrow 0$ when $n \rightarrow \infty$. for

We are going to prove that for each $\varepsilon>0$, there is $N>0$, such that for $n>N$, and

$$
L(0)^{n} \cdot S \lambda \in M(n) \cap \bar{D}_{r}
$$

we have

$$
\begin{gathered}
\left\|L(0)^{n} \cdot S \lambda-L(\lambda)^{n} u(\lambda)\right\|<\varepsilon / 2 \\
d\left(L(\lambda)^{n} u(\lambda), X_{r}(0)\right)<\varepsilon / 2,
\end{gathered}
$$


and as a consequence

$$
\begin{aligned}
& d\left(L(0)^{n} \cdot S \lambda, X_{r}(0)\right) \\
& \quad \leqq\left\|L(0)^{n} \cdot S \lambda-L(\lambda)^{n} u(\lambda)\right\|+d\left(L(\lambda)^{n} u(\lambda), X_{r}(0)\right)<\varepsilon .
\end{aligned}
$$

Let $y=L(0)^{n} \cdot S \lambda \in M(n) \cap \bar{D}_{r}$. Then $\lambda=S^{-1} L(0)^{-n} y$. So

$$
\|\lambda\|<\left\|S^{-1}\right\| \cdot\left\|L(0)^{-1}\right\|^{n} \cdot r \leqq C \mu_{2}^{n}(C \text { is a constant }) .
$$

Proof of (15). We have

$$
\begin{aligned}
& \left\|L(0)^{n} \cdot S \lambda-L(\lambda)^{n} u(\lambda)\right\| \\
& \quad \leqq\left\|L(0)^{n} \cdot S \lambda-L(0)^{n} u(\lambda)\right\|+\left\|L(0)^{n} u(\lambda)-L(\lambda)^{n} u(\lambda)\right\| .
\end{aligned}
$$

And by using Condition 3 and 4 and the inequality (18), we get

$$
\begin{aligned}
& \left\|L(0)^{n} \cdot S \lambda-L(0)^{n} u(\lambda)\right\| \leqq\|L(0)\|^{n} \cdot\|S \lambda-u(\lambda)\| \\
& \leqq C_{1} \mu_{1}^{n} \cdot\|\| \lambda \|^{2} \leqq C_{2} \mu_{1}^{n} \cdot \mu_{2}^{2 n}=C_{2} \mu^{n}, \\
& \left\|L(0)^{n} u(\lambda)-L(\lambda)^{n} u(\lambda)\right\| \\
& =\left\|\sum_{i=0}^{n-1}\left(L(0)^{n-i} L(\lambda)^{i}-L(0)^{n-i-1} L(\lambda)^{i+1}\right) u(\lambda)\right\| \\
& \leqq \sum_{i=0}^{n-1}\|L(0)\|^{n-i-1}\|L(\lambda)\|^{i}\|L(\lambda)-L(0)\| \cdot\|u(\lambda)\| \\
& \leqq C_{3} n \mu_{1}^{n-1}\|\lambda\|^{2} \leqq C_{4} n \mu_{1}^{n-1} \mu_{2}^{2 n}=C_{5} n \mu^{n},
\end{aligned}
$$

where $C_{i}$ are constants. Since $\lim _{n \rightarrow \infty} \mu^{n}=\lim _{n \rightarrow \infty} n \mu^{n}=0$, from (19), (20), (21), we get (15). Proof of (16). From (15) we have

$$
\left\|L(\lambda)^{n} u(\lambda)\right\|<r+\varepsilon / 2 .
$$

Choose $\varepsilon$ sufficiently small such that $r+\varepsilon<r^{\prime}$. Suppose $\lambda \in M$. We have $u(\lambda) \in X(\lambda)$ hence

$$
L(\lambda)^{n} u(\lambda) \in X(\lambda)
$$

If $L(\lambda)^{n} u(\lambda) \in V_{r}(\varepsilon / 2)$ [recall that $V_{r}(\varepsilon / 2)$ is the $\varepsilon / 2$-neighborhood of $\partial D_{r}$ ], then (16) is true. Otherwise

$$
L(\lambda)^{n} u(\lambda) \in X_{r}(\lambda) .
$$

According to part a) $\delta\left(X_{r}(\lambda), X_{r}(0)\right) \rightarrow 0$ when $\lambda \rightarrow 0$. So to prove (16) we need to show $\lambda \rightarrow 0$ when $n \rightarrow \infty$. But this is guaranteed by (18).

From (15), (16), we get (17). In other words: $\forall \varepsilon>0, \exists N>0$, such that $\forall n>N$, $\forall y \in M_{r}(n)$, we have $d\left(y, X_{r}(0)\right)<\varepsilon$, hence $\delta\left(M_{r}(n), X_{r}(0)\right) \rightarrow 0$ when $n \rightarrow \infty$.

b2) $\delta\left(X_{r}(0), M_{r}(n)\right) \rightarrow 0$ when $n \rightarrow \infty$.

b2.1) Let us prove at first that for each $x \in A \cap \bar{D}_{r}$ we have $d\left(x, M_{r}(n)\right) \rightarrow 0$. By Condition 2, there is a continuous mapping $h_{x}: \Delta \rightarrow E$ with $h_{x}(0)=x$ and $h_{x}(\lambda) \in X(\lambda)$. Lemma 4.3 below will show that fixing any neighborhood $\Delta^{\prime} \subset \Delta$ of 0 , there is $N^{\prime}>0$, such that for each $n>N^{\prime}$, the next equation has at least one solution in $\Delta^{\prime}$ :

$$
u(\lambda)-L(\lambda)^{-n} h_{x}(\lambda)=0
$$


i.e. there is $\lambda_{n} \in \Delta^{\prime}$, such that

$$
h_{x}\left(\lambda_{n}\right)=L\left(\lambda_{n}\right)^{n} u\left(\lambda_{n}\right) .
$$

Now we have automatically $\lambda_{n} \in M$, and hence $L(0)^{n} S \lambda_{n} \in M(n)$. Therefore

$$
\begin{gathered}
d\left(x, M_{r}(n)\right) \leqq d(x, M(n)) \leqq\left\|x-L(0)^{n} \cdot S \lambda_{n}\right\| \\
\leqq\left\|x-h_{x}\left(\lambda_{n}\right)\right\|+\left\|L\left(\lambda_{n}\right)^{n} u\left(\lambda_{n}\right)-L(0)^{n} \cdot S \lambda_{n}\right\|=I_{1}(n)+I_{2}(n) .
\end{gathered}
$$

Let us prove at first

$$
I_{2}(n)=\left\|L\left(\lambda_{n}\right)^{n} u\left(\lambda_{n}\right)-L(0)^{n} \cdot S \lambda_{n}\right\| \rightarrow 0 \quad \text { when } \quad n \rightarrow \infty .
$$

Since $L\left(\lambda_{n}\right)^{n} u\left(\lambda_{n}\right)=h_{x}\left(\lambda_{n}\right)$, and $h_{x}$ is continuous, we can suppose

$$
\left\|h_{x}\left(\lambda_{n}\right)\right\| \leqq \sup _{\lambda \in \Delta^{\prime}}\left\|h_{x}(\lambda)\right\|<r_{1}<r^{\prime}
$$

for some $r_{1}>0$. Then

$$
\left\|u\left(\lambda_{n}\right)\right\|=\left\|L\left(\lambda_{n}\right)^{-n} h_{x}\left(\lambda_{n}\right)\right\| \leqq r_{1}\left\|L\left(\lambda_{n}\right)^{-1}\right\|^{n} \leqq r_{1} \mu_{2}^{n} .
$$

By Condition 4 of the proposition, we have also

$$
\|S\| \cdot\|\lambda\| \leqq\|u(\lambda)\|+C\|\lambda\|^{2} .
$$

Take $\alpha<\|S\| C^{-1}$, then for $\|\lambda\| \leqq \alpha$, we have

$$
\|S\| \cdot\|\lambda\| \leqq\|u(\lambda)\|+C \alpha\|\lambda\| .
$$

Hence

$$
\|\lambda\| \leqq C_{0}\|u(\lambda)\|
$$

Combining with (25), we get

$$
\left\|\lambda_{n}\right\| \leqq C^{\prime} \mu_{2}^{n}
$$

(C, $C_{0}, C^{\prime}$ are constants). Hence for $\Delta^{\prime} C D(0, \alpha)$, the solutions $\lambda_{n}$ of (23) also verify (18). By using the results of (19), (20), (21) we get that for $\varepsilon>0$ and $n>\max \left\{N, N^{\prime}\right\}$, the formula (15) holds for $\lambda_{n}$, i.e.. $I_{2}(n)<\varepsilon / 2$.

From the continuity of $h_{x}$ and (27), we may also assume that under the same condition for $n$,

$$
I_{1}(n)=\left\|x-h_{x}\left(\lambda_{n}\right)\right\|<\varepsilon / 2 .
$$

Hence $I_{1}(n)+I_{2}(n) \rightarrow 0$ when $n \rightarrow \infty$. From (24) we get finallly

$$
d\left(x, M_{r}(n)\right) \rightarrow 0 \quad \text { when } n \rightarrow \infty \text {. }
$$

b2.2) For the general case, let us do as in a). For each $\varepsilon>0$, choose $\left\{x_{i}\right\}$ a finite subset of $A \cap \bar{D}_{r}$ such that

$$
X_{r}(0) \subset V_{r}(\varepsilon / 2) \cup \bigcup_{i} D\left(x_{i}, \varepsilon / 2\right),
$$

and choose $n$ large enough such that $\forall x \in X_{r}(0)$, we have

$$
d\left(x, M_{r}(n)\right) \leqq\left\|x-x_{i}\right\|+d\left(x_{i}, M_{r}(n)\right)<\varepsilon
$$


for some $i$. Hence

$$
\delta\left(X_{r}(0), M_{r}(n)\right) \rightarrow 0
$$

when $n \rightarrow \infty$.

c) This is a direct result from part b), by taking $0<s<r /\|S\|$.

The lemma below we needed in the proof of the theorem is in fact a topological result, which says that under small perturbations a continuous mapping does not lose zeros.

Lemma 4.3. Let $\Delta^{\prime} C E$ be a neighborhood of 0 , and $u: \Delta^{\prime} \rightarrow E$ be continuous, $u(0)=0$, $T_{0} u=S$ exists and is non-singular. Then there is $\eta>0$ such for any $w: \Delta^{\prime} \rightarrow E$ continuous with $\|w(x)\|<\eta$, the mapping $u+w$ has at least one zero in $\Delta^{\prime}$.

Proof. There is $\varepsilon>0$, such that $S_{\varepsilon}=\{x \in E \mid\|x\|=\varepsilon\}$ is contained in $\Delta^{\prime}$, the set $u\left(S_{\varepsilon}\right)$ does not contain 0 and the induced mapping

$$
u_{*}: H_{k-1}\left(S_{\varepsilon}\right) \rightarrow H_{k-1}(E-\{0\})=\mathbb{Z}
$$

is an isomorphism (where $k$ is the real dimension of $E$ and $H_{j}$ is the $j^{\text {th }}$ homology group). Let

$$
\eta=\inf \left\{\left.\|u\|\right|_{u \in S_{\varepsilon}}\right\}
$$

Then for $w: \Delta \rightarrow E$ continuous and $\|w(x)\|<\eta$, we have $u+w: S_{\varepsilon} \rightarrow E-\{0\}$, and the induced mapping $(u+w)_{*}$ is also an isomorphism, since $u+t w$ gives a homotopy between $u$ and $u+w$. We claim then $u+w$ has zeros in $\bar{D}_{\varepsilon}=\{x \in E \mid\|x\| \leqq \varepsilon\}$. If not, $(u+w)_{*}: H_{k}\left(\bar{D}_{\varepsilon}\right) \rightarrow H_{k}(E-\{0\})=\mathbb{Z}$ would be trivial homomorphism, since $u+w$ is continuous on $\bar{D}_{\varepsilon}$, and $H_{k}\left(\bar{D}_{\varepsilon}\right)$ would be trivial. This is a contradiction.

To induce (22) from this lemma we choose $N$ such that if $n>N$ then

$$
\left\|\frac{h_{x}(\lambda)}{L(\lambda)^{n}}\right\|<\eta, \quad \forall \lambda \in \Delta^{\prime} .
$$

Remark. If $u$ and $w$ are $\mathbb{C}$-analytic, then under the same condition $u+w$ has a unique zero in $\bar{D}_{\varepsilon}$ for small $\varepsilon$.

\section{Similarity Between $M$ and $J_{c}$ for $c$ a Misiurewicz Point}

In this section we are going to apply the result of Sect. 4 to show the similarity between the Mandelbrot set $M$ and the Julia set $J_{c}$ for $c$ a Misiurewicz point.

Recall that under $f_{c}: z \mapsto z^{2}+c$ the two sets $K_{c}, J_{c}$ are completely invariant. Hence $0 \in K_{c}$ if and only if $c=f_{c}(0) \in K_{c}$. We can then express the form of the Mandelbrot set by

$$
M=\left\{c \in \mathbb{C} \mid c \in K_{c}\right\} .
$$

Recall that $c \in \mathbb{C}$ is a Misiurewicz point if 0 is eventually periodic for $f_{c}$ but not periodic, and in this case $K_{c}=J_{c}$. According to Corollary 3.5, $J_{c}$ is asymptotically self-similar about $c$ with a scale $\varrho$ and with a certain limit model $Z$. We will prove that $M$ is also asymptotically $\varrho$-self-similar about $c$ but with the limit model $\lambda Z$, for a certain complex number $\lambda$. In other words, 
1. $M$ is asymptotically self-similar about $c$;

2. $M$ and $J_{c}$ are asymptotically similar about $c$, up to a multiplication by a complex number.

Or to be more explicit:

Theorem 5.1. Let $c_{0}$ be a Misiurewicz point. Then there are $\varrho_{0}$ with $\left|\varrho_{0}\right|>1, r, r^{\prime}$, $s>0$, a closed set $Z \subset \mathbb{C}$ with $\varrho_{0} Z=Z$, and $\lambda \in \mathbb{C}-\{0\}$ such that

$$
\begin{gathered}
\lim _{n \rightarrow \infty}\left(\varrho_{0}^{n} \tau_{-c_{0}} J_{c_{0}}\right)_{r}=Z_{r}, \\
\lim _{n \rightarrow \infty}\left(\varrho_{0}^{n} \tau_{-c_{0}} M\right)_{r^{\prime}}=(\lambda Z)_{r^{\prime}},
\end{gathered}
$$

and

$$
\lim _{t \in \mathbb{C}, t \rightarrow \infty} d_{s}\left(t \tau_{-c_{0}} M, t \lambda \cdot \tau_{-c_{0}} J_{c_{0}}\right)=0 .
$$

The precise form of $\varrho_{0}, \lambda, Z$ will be given in (32), (37), and (36).

Proof. We will apply Proposition 4.1. Since $K_{c}$ and $J_{c}$ are not self-similar about $c$, but only asymptotically self-similar, we have to adapt the situation at first.

Let $l$ be the minimal number such that $f_{c_{0}}^{l}\left(c_{0}\right)$ is periodic and let $p$ be the period of $f_{c_{0}}^{l}\left(c_{0}\right)$. Set $\left.\alpha_{0}=f_{c_{0}}^{l}\left(c_{0}\right)\right)$ and

$$
\varrho_{0}=\left(f_{c_{0}}^{p}\right)^{\prime}\left(\alpha_{0}\right) .
$$

We have $\left|\varrho_{0}\right|>1$ and $\left(f_{c_{0}}^{l}\right)^{\prime}\left(c_{0}\right) \neq 0$ and $J_{c_{0}}=K_{c_{0}}$. According to Theorem 3.2 the invariant set $K_{c_{0}}$ is asymptotically $\varrho\left(c_{0}\right)$-self-similar about $\alpha_{0}$, with a certain limit model $X\left(c_{0}\right)$, and about $c_{0}$ with the limit model $Z=\frac{1}{\left(f_{c_{0}}^{l}\right)^{\prime}\left(c_{0}\right)} X\left(c_{0}\right)$.

Since $\alpha_{0}$ is a repulsive periodic point of $f_{c_{0}}$, it is "stable" with respect to the parameter. In fact, applying twice the implicit function theorem, we can find a neighborhood $W$ of $c_{0}$ in $\mathbb{C}$ and two analytic functions

$$
\begin{array}{ll}
\alpha: W \rightarrow \mathbb{C}, & c \mapsto \alpha(c), \\
v: W \rightarrow \mathbb{C}, & c \mapsto v(c),
\end{array}
$$

such that

$$
\begin{gathered}
\alpha\left(c_{0}\right)=\alpha_{0}, \quad v\left(c_{0}\right)=c_{0}, \\
f_{c}^{l}(v(c))=\alpha(c), \quad f_{c}^{p}(\alpha(c))=\alpha(c), \quad\left(f_{c}^{l}\right)^{\prime}(v(c)) \neq 0,
\end{gathered}
$$

and $\varrho: c \mapsto \varrho(c)=\left(f_{c}^{p}\right)^{\prime}(\alpha(c))$ is analytic in $W$ with $|\varrho(c)|>1$ for all $c \in W$. According to Theorem 3.2 the filled-in Julia set $K_{c}$ is asymptotically $\varrho(c)$-self-similar about $\alpha(c)$ with a certain limit model $X(c)$, and about $v(c)$ with the limit model $\frac{1}{\left(f_{c}^{l}\right)^{\prime}(v(c))} X(c)$.

We need now an extension "with parameter" of Lemma 3.3:

Lemma 5.2 (high dimension case of Lemma 3.3). Suppose that $U$ is a neighborhood of $\left(\lambda_{0}, \alpha_{0}\right)$ in $\mathbb{C}^{n+1}=\mathbb{C}^{n} \times \mathbb{C}$. Let $G: U \rightarrow \mathbb{C}^{n+1}$ be a holomorphic function with $G(\lambda, z)=\left(\lambda, g_{\lambda}(z)\right)$ and $G\left(\lambda_{0}, \alpha_{0}\right)=\left(\lambda_{0}, \alpha_{0}\right)$. Assume $\left|g_{\lambda_{0}}^{\prime}\left(\alpha_{0}\right)\right|>1$. Then the sequence of mappings

$$
\Phi_{n}:(\lambda, z) \mapsto\left(\lambda,\left(g_{\lambda}^{\prime}(\alpha(\lambda))\right)^{n}\left(\left(g_{\lambda}^{-1}\right)^{n}(z)-\alpha(\lambda)\right)\right), \quad n=1,2, \ldots
$$


converges to a holomorphic mapping $\Phi: U_{1} \rightarrow \mathbb{C}^{n+1}$, where $U_{1} \subset U$ is a neighborhood of $\left(\lambda_{0}, \alpha_{0}\right), \alpha(\lambda)$ is the implicit holomorphic solution of $g_{\lambda}(z)=z$ satisfying $\alpha\left(\lambda_{0}\right)=\alpha_{0}$ and $g_{\lambda}^{-1}$ is the local inverse of $g_{\lambda}$. The mapping $\Phi$ has the following form

$$
\Phi(\lambda, z)=\left(\lambda, \varphi_{\lambda}(z)\right)
$$

with $\varphi_{\lambda}(\alpha(\lambda))=0, \varphi_{\lambda}^{\prime}(\alpha(\lambda))=1$ and

$$
\varphi_{\lambda}\left(g_{\lambda}(z)\right)=g_{\lambda}^{\prime}(\alpha(\lambda)) \cdot \varphi_{\lambda}(z) .
$$

According to this lemma the linearization mapping $\varphi_{c}$ of $f_{c}$ about $\alpha(c)$ varies analytically with respect to $c$. In other words, the mapping

$$
\phi:(c, z) \mapsto\left(c, \varphi_{c}(z)\right), \quad\left(c_{0}, \alpha_{0}\right) \mapsto\left(c_{0}, 0\right)
$$

is analytic in a neighborhood of $\left(c_{0}, \alpha_{0}\right)$. Since $(c, x) \mapsto\left(c, f_{c}^{l}(x)\right)$ is also analytic, the mapping

$$
\Phi:(c, z) \mapsto\left(c, \varphi_{c} \circ f_{c}^{l}(z)\right), \quad\left(c_{0}, c_{0}\right) \mapsto\left(c_{0}, 0\right)
$$

is analytic in a neighborhood $U$ of $\left(c_{0}, c_{0}\right)$.

Choose $r>0$ and restrict $W$ if necessary such that $W$ is closed and

$$
\Phi(c, c) \in \bar{D}_{r} \times W \subset \Phi(U) .
$$

Set consequently

$$
\begin{gathered}
\Omega=\Phi^{-1}\left(W \times \bar{D}_{r}\right), \\
\Omega_{c}=\{z \in \mathbb{C} \mid(c, z) \in \Omega\}, \\
K=\left\{(c, z) \mid z \in K_{c}\right\}, \\
X(c)=\varphi_{c} \circ f_{c}^{l}\left(K_{c} \cap \Omega_{c}\right),
\end{gathered}
$$

and

$$
X=\Phi(K \cap \Omega)=\{(c, x) \mid c \in W, x \in X(c)\} .
$$

Then $\Omega$ is closed in $\mathbb{C} \times \mathbb{C}, \Omega_{c}$ is closed for each $c \in W$, and

$$
c \in \Omega_{c} \text { for each } c \in W .
$$

Moreover, we possess an important property of $K$ proved by Douady and Hubbard:

Proposition 5.2 ([DH1], exposé no. VIII). The set $K$ is closed in $\mathbb{C} \times \mathbb{C}$.

Now we are ready to apply Proposition 4.1 to $X \subset W \times \mathbb{C}$. Set $E=\mathbb{C}, \Delta=W$,

$$
u: W \rightarrow \mathbb{C}, \quad c \mapsto \varphi_{c}\left(f_{c}^{l}(c)\right)
$$

and

$$
M_{u}=\{c \in W \mid u(c) \in X(c)\}
$$

Then $u\left(c_{0}\right)=0$ and

$$
M_{u}=\left\{c \in W \mid \varphi_{c} \circ f_{c}^{l}(c) \in \varphi_{c} \circ f_{c}^{l}\left(K_{c} \cap \Omega_{c}\right)\right\} .
$$

But from (33) we have $c \in \Omega_{c}$ as soon as $c \in W$, so

$$
M_{u}=\left\{c \in W \mid c \in K_{c} \cap \Omega_{c}\right\}=\left\{c \in W \mid c \in K_{c}\right\}=M \cap W .
$$


Hence $M_{u}$ is exactly the Mandelbrot set $M$ in a neighborhood of $c_{0}$. Let us checck the conditions of Proposition 4.1 now:

For Condition 1, since $K$ is closed (Proposition 5.3), $\Omega$ is closed, $\Phi$ is analytic so the set $X=\Phi(K \cap \Omega)$ is closed in $\mathbb{C} \times \mathbb{C}$ as well.

For Condition 2, we have at first $J_{c_{0}}=K_{c_{0}}$, that repulsive periodic points are dense in $J_{c_{0}}$, and that each repulsive periodic point $x$ is "stable" with respect to $c$. In fact, by the implicit function theorem, there is a neighborhood $U_{x}$ of $c_{0}$, an analytic mapping $h_{x}: U_{x} \rightarrow \mathbb{C}$ with $h_{x}\left(c_{0}\right)=x$ and $h_{x}(c)$ a repulsive periodic point of $f_{c}$. Hence $h_{x}(c) \in J_{c} \subset K_{c}$. Hence there is a dense set of continuous sections in $K$ at $\left(c_{0}, c_{0}\right)$, so does the set $X$ at $\left(c_{0}, 0\right)$. This gives Condition 2 for $X$.

For Condition 3, set $L(c)=\varrho(c) I$. Then $X(c)$ is $L(c)$-self-similar at 0 , and

$$
\begin{gathered}
\left\|L(c)-L\left(c_{0}\right)\right\|=\left|\varrho(c)-\varrho\left(c_{0}\right)\right|=O\left(\left|c-c_{0}\right|\right), \\
\left\|L\left(c_{0}\right)\right\| \cdot\left\|L\left(c_{0}\right)^{-1}\right\|^{2}=\left|\varrho_{0}\right| \cdot\left|\varrho_{0}\right|^{-2}=\left|\varrho_{0}\right|^{-1}<1 .
\end{gathered}
$$

Condition 3 is then well verified.

For Condition 4, since $u$ is analytic, Eq. (13) is automatically verified. To check $u^{\prime}\left(c_{0}\right) \neq 0$ is the main difficulty here. We will do that in Lemma 5.4 below, by using a non-trivial result of Douady and Hubbard. Moreover, we will get the following explicit form of $u^{\prime}\left(c_{0}\right)$ :

$$
u^{\prime}\left(c_{0}\right)=\left.\frac{d}{d c}\left(\varphi_{c}\left(f_{c}^{l}(c)\right)\right)\right|_{c=c_{0}}=\left.\frac{d}{d c}\left(f_{c}^{l}(c)\right)\right|_{c=c_{0}}-\left.\frac{d}{d c}(\alpha(c))\right|_{c=c_{0}} .
$$

Hence all the conditions of Proposition 4.1 are verified. From (14) we can claim then that $M \cap W=M_{u}$ is $\varrho\left(c_{0}\right)$-self-similar about $c_{0}$ and the limit model is $\frac{1}{u^{\prime}\left(c_{0}\right)} X\left(c_{0}\right)$, i.e. there is $r>0$ such that

$$
\lim _{n \rightarrow \infty}\left(\varrho\left(c_{0}\right)^{n} \tau_{-c_{0}} M\right)_{r}=\left(\frac{1}{u^{\prime}\left(c_{0}\right)} X\left(c_{0}\right)\right)_{r} .
$$

Recall that $X\left(c_{0}\right)=\varphi_{c_{0}} \circ f_{c_{0}}^{l}\left(K_{c_{0}}\right)$ is the limit model of $K_{c_{0}}=J_{c_{0}}$ at $\alpha\left(c_{0}\right)=f_{c_{0}}^{l}\left(c_{0}\right)$, and $\frac{1}{\left(f_{c_{0}}^{l}\right)^{\prime}\left(c_{0}\right)} X\left(c_{0}\right)$ is the limit model of $K_{c_{0}}$ at $c_{0}$. In other words, set

$$
Z=\frac{1}{\left(f_{c_{0}}^{l}\right)^{\prime}\left(c_{0}\right)} X\left(c_{0}\right)=\frac{1}{\left(f_{c_{0}}^{l}\right)^{\prime}\left(c_{0}\right)} \varphi_{c_{0}} \circ f_{c_{0}}^{l}\left(K_{c_{0}}\right)=\frac{1}{\left(f_{c_{0}}^{l}\right)^{\prime}\left(c_{0}\right)} \varphi_{c_{0}}\left(K_{c_{0}}\right)
$$

we have:

$$
\lim _{n \rightarrow \infty}\left(\varrho_{0}^{n} \tau_{-c_{0}} J_{c_{0}}\right)_{r}=Z_{r}
$$

That is (29). In (35) substitute $u^{\prime}\left(c_{0}\right)$ by its explicit form (34) and $X\left(c_{0}\right)$ by $\left(f_{c_{0}}^{l}\right)^{\prime}\left(c_{0}\right) Z$, we get finally (probably for a $r^{\prime}<r$ ):

$$
\lim _{n \rightarrow \infty}\left(\varrho\left(c_{0}\right)^{n} \tau_{-c_{0}} M\right)_{r^{\prime}}=\left(\frac{\left(f_{c_{0}}^{l}\right)^{\prime}\left(c_{0}\right)}{\left.\frac{d}{d c}\left(f_{c}^{l}(c)\right)\right|_{c=c_{0}}-\left.\frac{d}{d c}(\alpha(c))\right|_{c=c_{0}}} Z\right)_{r^{\prime}} .
$$

This is Eq. (30) of the theorem, with

$$
\lambda=\frac{\left(f_{c_{0}}^{l}\right)^{\prime}\left(c_{0}\right)}{\left.\frac{d}{d c}\left(f_{c}^{l}(c)\right)\right|_{c=c_{0}}-\left.\frac{d}{d c}(\alpha(c))\right|_{c=c_{0}}} .
$$


Equation (31) is a direct consequence of (30) and (29) according to Proposition 2.2.

Lemma 5.4. For $u: W \rightarrow \mathbb{C}, c \mapsto \varphi_{c}\left(f_{c}^{l}(c)\right)$, we have

$$
u^{\prime}\left(c_{0}\right)=\left.\frac{d}{d c}\left(f_{c}^{l}(c)\right)\right|_{c=c_{0}}-\left.\frac{d}{d c}(\alpha(c))\right|_{c=c_{0}}
$$

and

$$
u^{\prime}\left(c_{0}\right) \neq 0 .
$$

Proof. Set $\beta: \mathbb{C} \rightarrow \mathbb{C}, c \mapsto f_{c}^{l}(c)=f_{c}^{l+1}(0)$ and $w: \mathbb{C} \rightarrow \mathbb{C}, c \mapsto f_{c}^{l+1+p}(0)-f_{c}^{l+1}(0)$. We have $\beta\left(c_{0}\right)=\alpha_{0}$ and $w\left(c_{0}\right)=0$. We will see

$$
u^{\prime}\left(c_{0}\right)=\beta^{\prime}\left(c_{0}\right)-\alpha^{\prime}\left(c_{0}\right)=\frac{1}{\varrho\left(c_{0}\right)-1} w^{\prime}\left(c_{0}\right)
$$

and $w^{\prime}\left(c_{0}\right) \neq 0$.

Set $F(c, z)=\varphi_{c}(z)$, where $\varphi_{c}$ is the linearization mapping of $f_{c}$ about $\alpha(c)$. Then $u(c)=F(c, \beta(c))$ and

Since

$$
u^{\prime}\left(c_{0}\right)=\left.\frac{\partial F}{\partial c}\right|_{\left(c_{0}, \alpha_{0}\right)}+\left.\frac{\partial F}{\partial z}\right|_{\left(c_{0}, \alpha_{0}\right)} \cdot \beta^{\prime}\left(c_{0}\right)
$$

$$
\begin{gathered}
\left.\frac{\partial F}{\partial z}\right|_{\left(c_{0}, \alpha_{0}\right)}=\varphi_{c_{0}}^{\prime}\left(\alpha_{0}\right)=1, \\
\left.\frac{\partial F}{\partial c}\right|_{\left(c_{0}, \alpha_{0}\right)}=\lim _{c \rightarrow c_{0}} \frac{F\left(c, \alpha_{0}\right)-F\left(c_{0}, \alpha_{0}\right)}{c-c_{0}}=\lim _{c \rightarrow c_{0}} \frac{F\left(c, \alpha_{0}\right)-F(c, \alpha(c))}{c-c_{0}} \\
=-\left.\frac{\partial F}{\partial z}\right|_{\left(c_{0}, \alpha_{0}\right)} \cdot \alpha^{\prime}\left(c_{0}\right)=-\alpha^{\prime}\left(c_{0}\right)
\end{gathered}
$$

[since $F\left(c_{0}, \alpha_{0}\right)=F(c, \alpha(c))=0$ ], we have then $u^{\prime}\left(c_{0}\right)=\beta^{\prime}\left(c_{0}\right)-\alpha^{\prime}\left(c_{0}\right)$;

$$
\begin{aligned}
& w(c)=f_{c}^{p}\left(f_{c}^{l+1}(0)\right)-f_{c}^{p}(\alpha(c))+\alpha(c)-f_{c}^{l+1}(0), \\
& \lim _{c \rightarrow c_{0}} \frac{w(c)-w\left(c_{0}\right)}{c-c_{0}}=\lim _{c \rightarrow c_{0}} \frac{\beta(c)-\alpha(c)}{c-c_{0}}\left(\frac{f_{c}^{p}(\beta(c))-f_{c}^{p}(\alpha(c))}{\beta(c)-\alpha(c)}-1\right) \\
& =\left(\beta^{\prime}\left(c_{0}\right)-\alpha^{\prime}\left(c_{0}\right)\right)\left(\varrho\left(c_{0}\right)-1\right) .
\end{aligned}
$$

The fact $w^{\prime}\left(c_{0}\right) \neq 0$ is not evident and is proved by Douady and Hubbard. They have in fact several proofs about that. One is stated in the last corollary of [DH1], another one is an arithmetic method and is inserted in [DH3] (Lemma 1 of Chapter V).

Theorem 5.5 (Review). Let us summarize now the main steps to prove the similarity between $M$ and $J_{c}$. That is, the main steps of the adaptation of the Misiurewicz case to the assumptions of Proposition 4.1.

Assume that $c_{0}$ is eventually repulsive periodic under the iteration of $f_{c_{0}}$ (i.e. $c_{0}$ is a Misiurewicz point $)$. Let $l, p$ be the minimal integers such that $f_{c_{0}}^{p}\left(f_{c_{0}}^{l}\left(c_{0}\right)\right)=f_{c_{0}}^{l}\left(c_{0}\right)$. Set $\alpha_{0}=f_{c_{0}}^{l}\left(c_{0}\right)$ and $\varrho_{0}=\left(f_{c_{0}}^{p}{ }^{\prime}\left(\alpha_{0}\right)\right.$. Then for $c$ varying in a neighborhood $W$ of $c_{0}$ in $\mathbb{C}$ : 
1. There is a unique repulsive periodic point $\alpha(c)$ of period $p$ for $f_{c}$ such that $c \mapsto \alpha(c)$ is analytic and $\alpha\left(c_{0}\right)=\alpha_{0}$; let $\varrho(c)=\left(f_{c}^{p}\right)^{\prime}(\alpha(c))$, the mapping $c \mapsto \varrho(c)$ is analytic with $\varrho\left(c_{0}\right)=\varrho_{0}$ (implicit function theorem);

2. $K_{c}$ is asymptotically $\varrho(c)$-self-similar about $\alpha(c)$ with a certain limit model $X(c)$, provided that it is completely invariant under $f_{c}$ (Theorem 3.2 );

3. There exists a conformal mapping (the linearization mapping) $\varphi_{c}$ defined in a neighborhood of $\alpha(c)$ in $\mathbb{C}$ with $\varphi_{c}(\alpha(c))=0, \varphi_{c}^{\prime}(\alpha(c))=1, \varphi_{c}\left(K_{c}\right)=X(c)$, and $(c, z) \mapsto\left(c, \varphi_{c}(z)\right)$ is analytic in a neighborhood of $\left(c_{0}, \alpha_{0}\right)$ in $\mathbb{C} \times \mathbb{C}$ (Lemma 3.3, Theorem 3.2 and Lemma 5.2);

4. The set $K=\left\{(c, z) \mid z \in K_{c}\right\}$ is closed in $W \times \mathbb{C}$ (Proposition 5.3). This gives Condition 1 of Proposition 4.1;

5. $K_{c_{0}}=J_{c_{0}}$ (Proposition 3.1). This gives Condition 2 of Proposition 4.1, provided that repulsive periodic points are dense in $J_{c_{0}}$;

6. Condition 3 of Proposition 4.1 is a consequence of 2 and 3;

7. For $u: c \mapsto \varphi_{c}\left(f_{c}^{l}(c)\right)$, we have $M \cap W=\{c \in \mathbb{C} \mid u(c) \in X(c)\}=M_{u}$;

8. $u$ is analytic and $u^{\prime}\left(c_{0}\right) \neq 0$ (Lemma 5.4). This gives Condition 4 of Proposition 4.1;

9. $M$ is asymptotically $\varrho_{0}$-self-similar about $c_{0}$ with the limit model $\frac{1}{u^{\prime}\left(c_{0}\right)} X\left(c_{0}\right)$ (Proposition 4.1);

10. $\left(f_{c_{0}}^{l}\right)^{\prime}\left(c_{0}\right) \neq 0$ and $K_{c_{0}}$ about $c_{0}$ is asymptotically $\varrho_{0}$-self -similar, with the limit model $\frac{1}{\left(f_{c_{0}}^{l}\right)^{\prime}\left(c_{0}\right)} X\left(c_{0}\right)$ (Theorem 3.2);

11. Finally $M$ about $c_{0}$ and $K_{c_{0}}$ about $c_{0}$ are asymptotically similar, up to a multiplication by $\frac{\left(f_{c_{0}}^{l}\right)^{\prime}\left(c_{0}\right)}{u^{\prime}\left(c_{0}\right)}$ (consequence of 9 and 10 and Proposition 2.2).

To simplify the notation, denote by $X\left(c_{0}\right), Y\left(c_{0}\right)$ and $Z\left(c_{0}\right)$ the limit model of $K_{c_{0}}$ at $\alpha_{0}$, of $M$ at $c_{0}$ and of $K_{c_{0}}$ at $c_{0}$ respectively. We have $Y\left(c_{0}\right)=\frac{1}{u^{\prime}\left(c_{0}\right)} X\left(c_{0}\right)$ and $Z\left(c_{0}\right)=\frac{1}{\left(f_{c_{0}}^{l}\right)^{\prime}\left(c_{0}\right)} X\left(c_{0}\right)$

\section{Examples}

1. Two spirals. We see a lot of spirals in $M$. The point $c \approx-0.77568377$ $+0.13646737 i$ is a Misiurewicz point which is chosen so that $M$ has a two spiral shape near $c$. Figure $9 \mathrm{a}$ is a magnification of $J_{c}$ around $c$ and Fig. $9 \mathrm{~b}$ is a magnification of $M$ around $c$.

2. Triple point. The point $c=-0.1011+0.95629 i$ is the 3 -fold bifurcation point near the top of the Mandelbrot set. It is also a typical Misiurewicz point. In this case, $l=3, p=1$,

$$
\begin{gathered}
\alpha=(1 / 2)(1-\sqrt{1-4 c}) \approx-0.3276+0.57776 i, \\
\varrho=2 \alpha \approx-0.6552+1.15552 i, \\
u^{\prime}(c) \approx-4.7515+2.06497 i, \quad a=\left(f_{c}^{3}\right)^{\prime}(c) \approx-6.4464+0.1808 i .
\end{gathered}
$$

In other words: $|\varrho| \approx 1.328, \arg (\varrho) \approx 119.6^{\circ},\left|u^{\prime}(c)\right| \approx 5.1808, \arg \left(u^{\prime}(c)\right)=156.51^{\circ}$, $|a| \approx 6.4489$, and $\arg (a)=178.39^{\circ}$. Figures $10 \mathrm{a}$ are two successive magnifications of 

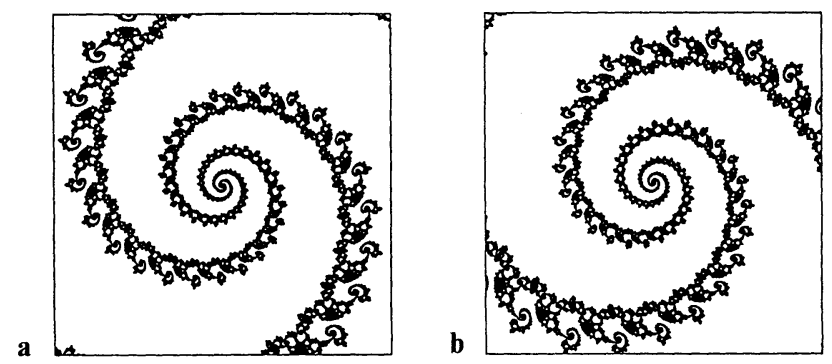

Fig. 9a, b. Magnifications of $J_{c}$ and of $M$ for $c=-0.77568377+0.13646737 i$. a $J_{c}$, center: $c$, width: 0.00018 . b $M$, center: $c$, width: 0.00024
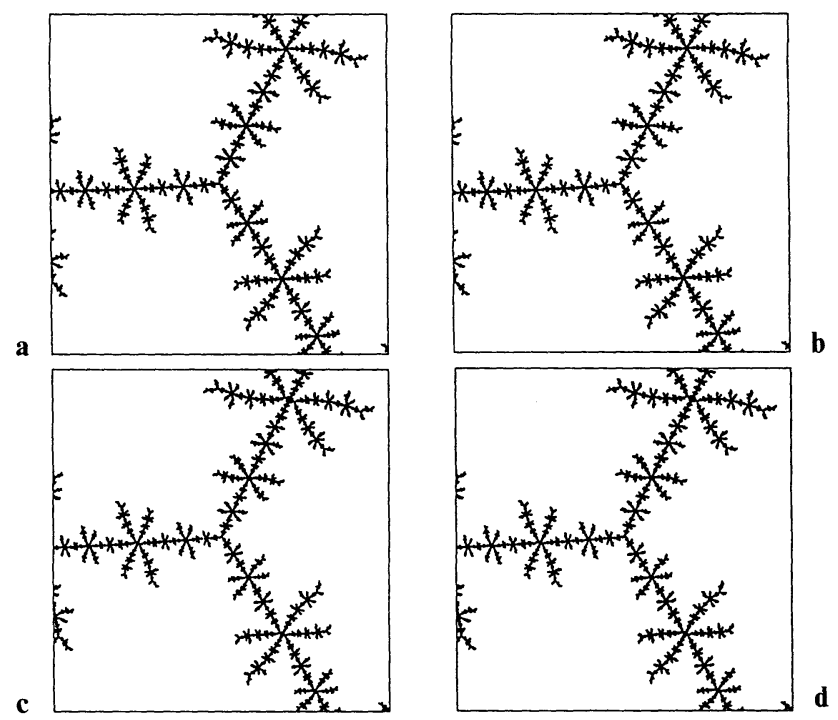

Fig. 10a-d. Magnifications of $J_{c}$ and $M$ around $c=-0.1011+0.9563 i$. a $J_{c}$, center: $\alpha$, width: 0.01 , rotation: $0 . \mathbf{b} J_{c}$, center: $\alpha$, width: 0.00753 , rotation: $119.6^{\circ}$. c $J_{c}$, center: $c$, width: 0.00155 , rotation: $178.39^{\circ}$. d $M$, center: $c$, width 0.00193 , rotation: $156.51^{\circ}$

$J_{c}$ centered at $\alpha$ with the magnification factors differed by $\varrho$. Figure $10 \mathrm{c}=Z(c)$ $=X(c) / \alpha$. Figure $10 \mathrm{~d}=Y(c)=X(c) / u^{\prime}(c)$. With these carefully chosen factors, we can not distinguish $M$ and $J_{c}$ any more.

3. Let us take the example of $c=i$ again. Recall that for $f_{i}: z \mapsto z^{2}+i$, we have $l=1$, $p=2, \alpha=i-1, \varrho=4 \sqrt{2} e^{\pi i / 4}$, and $\left(f_{c}^{l}\right)^{\prime}(c)=\left(f_{i}\right)^{\prime}(i)=2 i$. For the value of $\lambda$, we have

$$
\left.\frac{d}{d c}\left(f_{c}^{l}(c)\right)\right|_{c=i}=\left.\frac{d}{d c}\left(c^{2}+c\right)\right|_{c=i}=\left.(2 c+1)\right|_{c=i}=2 i+1 .
$$

The function $\alpha(c)$ is the solution near $i-1$ of the implicit equation

$$
f_{c}^{2}(z)=z, \text { i.e. }\left(z^{2}+c\right)^{2}+c-z=0
$$

for $c$ close to $i$. Hence

$$
\left.\frac{d}{d c}(\alpha(c))\right|_{c=i}=-\frac{2\left((i-1)^{2}+i\right)+1}{2\left((i-1)^{2}+i\right) \cdot 2(i-1)-1}=\frac{2 i-1}{4 i+3} .
$$



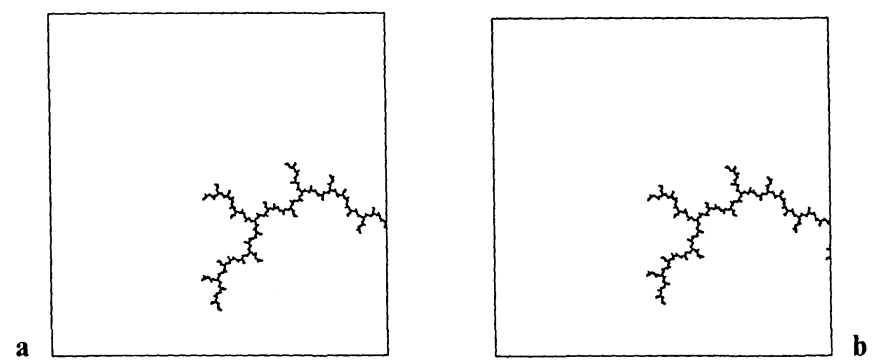

Fig. 11a, b. Magnifications of $J_{i}$ and $M$ around $i$. a $J_{i}$, center: $i$, width: 0.0001 , rotation: 0. b $M$, center: $i$, width: $0.0001 \times \sqrt{5} / 2$; rotation: $-26.565^{\circ}$

Then

$$
\lambda=\frac{2 i}{2 i+1-\frac{2 i-1}{4 i+3}}=1+\frac{1}{2} i .
$$

In other words, $|\lambda|=\sqrt{5} / 2 \approx 1.118$ and $\arg (\lambda) \approx 26^{\circ} 565$. Figure 11 shows the similarity between $M$ and $J_{i}$.

Figure 12 generated by Peitgen, Jürgens, and Sauper ([PJS]) shows the selfsimilarity of $M$ about $i$ in another way. By our result, the set $M$ should be almost unchanged if we make two powerful blowups centered at $i$ with the factors differed by $4 \sqrt{2}$ and by a rotation $\pi / 4$. If we just blow it up successively by $4 \sqrt{2}$ without rotating, each one would differ by a $\pi / 4$ rotation from the last one. Hence after eight magnifications, we would see almost the same set. Compare for example Fig. 12-4 and Fig. 12-12. Do you see any difference?

It seems that $M$ does not spiral at $i$. But that is our human eyes' mistake. The rotation angle $\pi / 4$ explains well that there is a spiral there. The reason that we can not "see" it is because the absolute value of $\varrho$ which is $4 \sqrt{2}$ is too large with respect to its angle $\pi / 4$, so that the spiral is "absorbed" by the center. For example, take a point $x$ on the positive real axis. Then $x / \varrho^{8}$ is again a positive real number but its value is decreased by a factor $1 / 2^{24}$ ! It is so close to 0 that human eyes cannot distinguish them any more. As an experiment, we take the spiral

$$
A(s)=\left\{e^{(\log s+\pi i / 4) x} \mid-\infty<x<+\infty\right\}
$$

which is blowing-up-invariant by the factor $\varrho=s e^{\pi i / 4}$. The two pictures in Fig. 5 $(s=1.2$ and $s=4 \sqrt{2}$ ) show how the spiral "disappears" as $s$ increases.

\section{Other Results}

To end this paper we give a rough description of some other similarity problems. We will see that for our definition of similarity the Misiurewicz points are the only interesting case.

6.1. Small Copies of $M$ Converging to a Misiurewicz Point. By looking at the Mandelbrot set one realizes easily that it contains a lot of copies of itself. Eckmann 

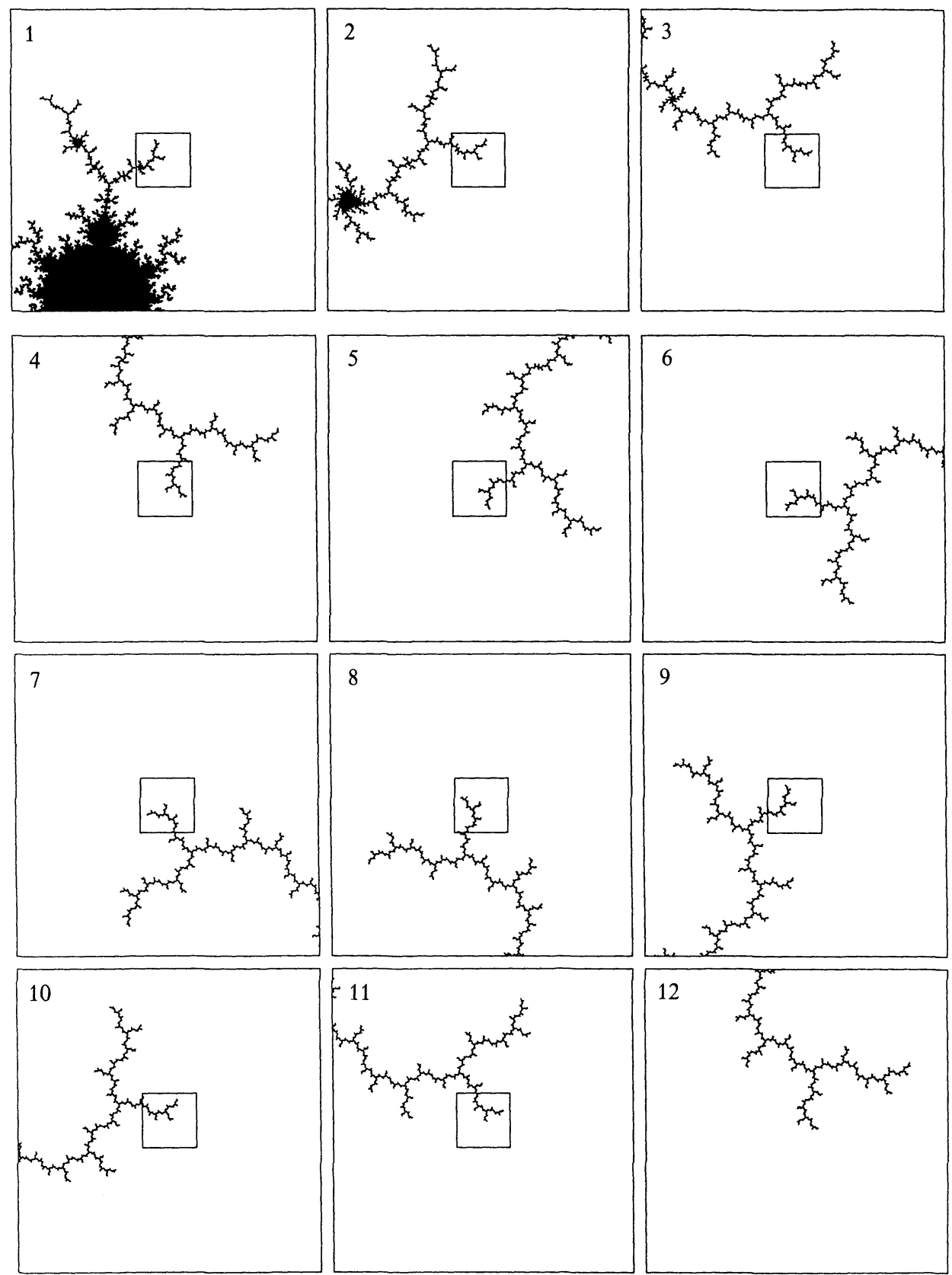

Fig. 12. 12 successive magnifications of $M$ as indicated by the inserted frames. Center: $i$. Width of the first picture: $0.5656854 \approx 0.1 \times 4 \sqrt{2}$. Magnification factor: $4 \sqrt{2}$ 
and Epstein ([EE]) and Douady and Hubbard ([DH3]) have studied the existence and the scaling property of small copies of $M$ near a Misiurewicz point. More precisely, for each Misiurewicz point $c \in M$, there is a sequence of copies $M_{n} \subset M$ of $M$ converging to $c$ geometrically with the ratio $1 / \varrho$ (where $\varrho$ is equal to our similarity scale for $M$ about $c$ ), and the diameter of $M_{n}$ decreases to 0 also geometrically, but with the ratio $1 /|\varrho|^{2}$. Hence $\operatorname{diam}\left(M_{n}\right)$ decreases faster than the convergence of $M_{n}$ to $c$. This is compatible with our result that the limit model of $M$ at $c$ has no interior point.

6.2. Some Trivial Cases. Using our definition of similarity one can check about other points too. But sometimes the situation is more or less trivial so that it might be better to analyze other notions of similarities.

Here is a list of the trivial cases:

1. Attractive periodic points in $K_{f}$ and hyperbolic components of $M$.

Suppose that $f$ is a monic polynomial and $x$ is an eventually (super)attractive periodic point. Then $x \in K_{f}$ and $K_{f}$ is self-similar about $x$. In fact $K_{f}$ is trivially selfsimilar about any point $y$ of $K_{f}$, since for $r$ small enough we have $\left(\tau_{-y} K_{f}\right)_{s}=\bar{D}_{s}$ for every $s<r$.

Similarly, the Mandelbrot set $M$ is trivially self-similar about any point $c \in M$. Specially, for $c$ in a hyperbolic component of $\dot{M}$ [i.e. $f_{c}$ has an (super)attractive period cycle], $M$ and $J_{c}$ are trivially similar about $c$.

2. Rational indifferent periodic points in $J_{f}$ and parabolic points in $M$.

First of all we observe that any two curves tangent at a point $x$ are asymptotically similar about $x$. Furthermore any closed set in $\mathbb{C}$ limited by two tangent curves is asymptotically self-similar about that point, with the common tangent line as the limit model.

Suppose that $x$ is a rational indifferent periodic point for $f_{c}$ (for example, $x=\alpha$ in Fig. 3d). Then $x \in J_{c}$ and there are finite petals in $\mathscr{K}_{c} \cup\{x\}$ located one-by-one tangently around $x([\mathrm{~B}, \mathrm{DH} 2])$. Hence the Julia set is limited by tangent curves and is asymptotically self-similar about $x$.

A point $c \in \mathbb{C}$ is called parabolic if $f_{c}$ has a rational indifferent cycle. Then $c \in \partial M$ and $c$ is in the boundary of either one or two hyperbolic components ([DH2]). If $c$ is on the boundary of two hyperbolic components, the two components are tangent at $c$ with tangent order two ([DH2, T1]), consequently $\partial M$ and $M$ are asymptotically self-similar about $c$. If $c$ is on the boundary of only one hyperbolic component $W$ of $M, \partial W$ has a cusp at $c([\mathrm{DH} 2])$ so $\partial M$ in a neighborhood of $c$ is again limited by tangent curves and then is asymptotically self-similar about $c$.

6.3. Feigenbaum Points. The Feigenbaum points form a different set of boundary points of $M$. The classical Feigenbaum point is the limit point of the period doubling sequence on the real axis starting at $c=-1$, which is first studied experimentally by Feigenbaum and Großmann and Thomae. According to a theory of Douady and Hubbard ([DH3]) $M$ contains infinitely many small copies of itself. Moreover, for each point $c \in M$ such that $f_{c}$ has a superattractive orbit, there is a homeomorphism

$$
\psi: M \rightarrow c \perp M \subset M
$$

with $\psi(0)=c$ and $\partial(c \perp M) \subset \partial M$. The point $c \perp x$ is called the "tuning" of $x$ by $c$. We can then consider the sequence $c, c \perp c, c \perp c \perp c, \ldots$ and see if it converges, i.e. if the infinite tuning exists. We call the eventual limit point a Feigenbaum point. For example the above classical Feigenbaum point corresponds to the infinite tuning 

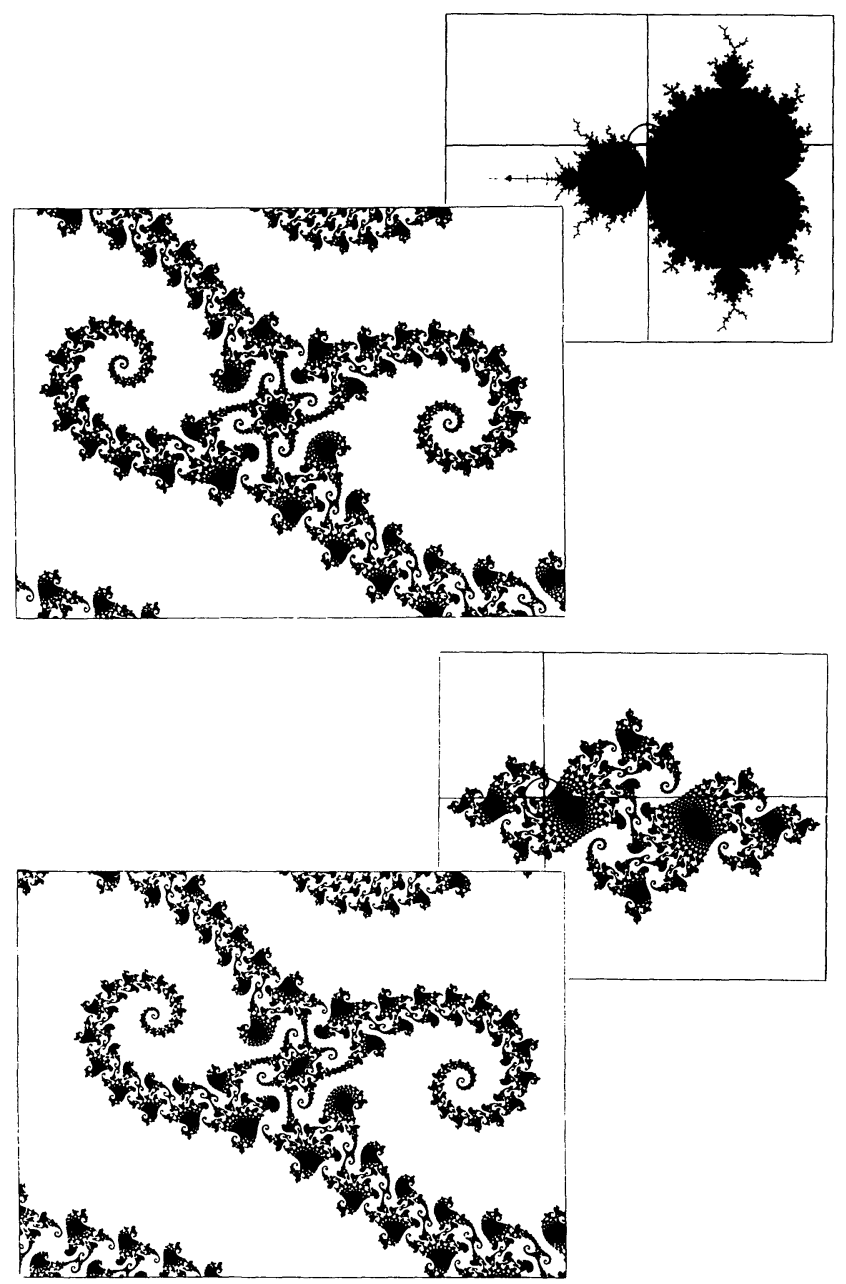

Fig. 13. $M$ and $J_{c}$ around $c$, where $c=-0.745429+0.113008 i$. See also [PS]

of $c=-1$. Feigenbaum has conjectured that the limit point for $c=-1$ exists and the sequence $c, c \perp c, c \perp c \perp c, \ldots$ converges geometrically. A more general conjecture is:

Conjecture 1. For every point $c$ such that $f_{c}$ has a superattractive orbit, the sequence

$$
c, c \perp c, c \perp c \perp c, \ldots
$$

converges geometrically.

Some special cases of the conjecture including the case $c=-1$ have been well studied and confirmed. Here is a list of references: [CE, CEL, EEW, EW, GSK, and L].

Denote by $c^{\perp \infty}$ the limit point and $\delta$ the inverse of the convergent-ratio. We have $|\delta|>1$. Milnor ([M]) has made some computer experiments on the local 
structure of $M$ about $c^{\perp \infty}$ and has suggested that $M$ should be asymptotically measurely self-similar at $c^{\perp \infty}$ with the scale $\delta$. In other words,

Conjecture 2. There is a measurable set $X$ such that $\left(\delta^{n}\left(\tau_{-c^{\perp \infty}} M\right)\right)_{r}$ converges for the Lebesgue measure to $X$. The set $X$ is not closed, is everywhere dense but may have small measure.

The fact that $X$ should not be closed excludes any application of the Hausdorff-Chabauty distance.

6.4. Peitgen's Observation. H.-O. Peitgen ([PS]) has observed experimentally another similarity phenomenon between $M$ and $K_{c}(F i g .13)$ : for some value of $c$ a magnification with a carefully chosen power (neither too small nor too large) of $M$ and $J_{c}$ about $c$ gives very similar images. This phenomenon is different from what we did in this paper because the means of similarity is rather local but not asymptotic.

\section{References}

[B] Blanchard, P.: Complex analytic dynamics on the Riemann sphere. Bull. Am. Soc. 11, 85-141 (1984)

[CE] Campanino, M., Epstein, H.: On the existence of Feigenbaum's fixed point. Commun. Math. Phys. 79, 226-302 (1981)

[CEL] Collet, P., Eckmann, J.-P., Lanford III, O.E.: Universal properties of maps on an interval. Commun. Math. Phys. 76, 211-254 (1980)

[D] Douady, A.: Système dynamiques holomorphes, exposé no. 599, séminaire Bourbaki 1982/83. Astérisque 105-106, 39-63 (1983)

[DH1] Douady, A., Hubbard, J.H.: Etude dynamique des polynômes complexes. Part I. Publication mathématique d'Orsay, 84-02, 1984

[DH2] Douady, A., Hubbard, J.H.: Etude dynamique des polynômes complexes. Part II. Publication mathématique d'Orsay, Orsay, 85-02, 1985

[DH3] Douady, A., Hubbard, J.H.: On the dynamics of polynomial-like mappings. Ann. Scient. Éc. Norm. Sup., $4^{e}$ série, t. 18, 287-343 (1985)

[EE] Eckmann, J.-P., Epstein, H.: Scaling of Mandelbrot sets generated by critical point preperiodicity. Commun. Math. Phys. 101, 283-289 (1985)

[EEW] Eckmann, J.-P., Epstein, H., Wittwer, P.: Fixed points of Feigenbaum's type for the equation $f^{p}(\lambda x) \equiv \lambda f(x)$. Commun. Math. Phys. 93, 495-516 (1984)

[EW] Eckmann, J.-P., Wittwer, P.: A complete proof of the Feigenbaum conjectures. J. Stat. Phys. 46, Nos. 3/4, 455-475 (1987)

[F1] Feigenbaum, M.: The universal metric properties of nonlinear transformations. J. Stat. Phys. 21, 669-706 (1979)

[F2] Feigenbaum, M.: Universal behavior in nonlinear systems. Los Alamos Sci. 1, 4-27 (1980)

[GSK] Gol'berg, A.I., Sinai, Ya.G., Khanin, K.M.: Universal pronerties fes , ances of bifurcations of period three. Russ. Math. Surv. 38, 187-188 (1983)

[GT] Großmann, S., Thomae, S.: Invariant distributions and stationary correlation functions of one-dimensional discrete processes. Z. Naturforsch. 32a, 1353-1363 (1977)

[L] Lanford III, O.E.: A computer-assisted proof of the Feigenbaum conjectures. Bull. (new series) Am. Soc., Vol. 6, Number 3, 427-434 (1982)

[M] Milnor, J.: Self-similarity and hairiness in the Mandelbrot set. In Computers in Geometry and Topology. Martin, C. (ed.). Tangora. New York, Basel: Dekker, pp. 211-257 1989

[PR] Peitgen, H.-O., Richter, P.H.: The beauty of fractals. Berlin, Heidelberg, New York: Springer 1986

[PS] Peitgen, H.-O., Sauper, D. (eds.): The science of fractal images. Berlin, Heidelberg, New York: Springer 1988 
[PJS] Peitgen, H.-O., Jürgens, H., Sauper, D.: The Mandelbrot set: a paradigm for experimental mathematics, ECM/87-Educational Computing in Mathematics, Banchoff, T.F. et al. (eds.), Elsevier Science Publishers BV. (North-Holland), 1988

[T1] Tan Lei: Ordre du contact des composantes hyperboliques de $M$, in [DH2], exposé no. XV

[T2] Tan Lei: Ressemblence entre l'ensemble de Mandelbrot et l'ensemble de Julia au voisinage d'un point de Misiurewicz, in [DH2], exposé no. XXIII

Communicated by J.-P. Eckmann 
